\title{
A Simulated Annealing Based Centre of Mass (SAC) Approach for Mesh Routers Placement in Rural Areas
}

\author{
Jean Louis Kedieng Ebongue Fendji, University of Ngaoundéré, Yaounde, Cameroon \\ (iD) https://orcid.org/0000-0001-9803-6981 \\ Chris Thron, Texas A\&M University-Central Texas, USA \\ iD https://orcid.org/0000-0002-8960-2504
}

\begin{abstract}
The problem of node placement in a rural wireless mesh network (RWMN) consists of determining router placement which minimizes the number of routers while providing good coverage of the area of interest. This problem is NP-hard with a factorial complexity. This article introduces a new approach, called the simulated annealing-based centre of mass (SAC) for solving this placement problem. The intent of this approach is to improve the robustness and the quality of solution, and to minimize the convergence time of a simulated annealing (SA) approach in solving the same problem in small and large scale. SAC is compared to the centre of mass (CM) and simulated annealing (SA) approaches. The performances of these algorithms were evaluated on a set of 24 instances. The experimental results show that the SAC approach provides the best robustness and solution quality, while decreasing by half the convergence time of the SA algorithm.
\end{abstract}

\section{KEYWORDS}

Centre of Mass, Convergence Time, Node Placement, Robustness, Simulated Annealing, Wireless Mesh Networks

\section{INTRODUCTION}

The ninth United Nations Sustainable Development Goal is to build resilient infrastructure. One of his targets is to significantly increase access to ICT and strive to provide universal and affordable access to internet in Least Developed Countries by 2020. Universal and affordable access means "Leave No One Behind". But the bulk of the unconnected is located in rural and hard-to-wire areas, with technical and financial limitations. Fortunately, Wireless Mesh Network (WMN) (Akyildiz, Wang, \& Wang, 2005) has appeared as an appealing cost-effective solution to bridge the digital divide and to connect the unconnected. A Wireless Mesh Network (WMN) is a wireless network in which nodes are connected in a mesh topology. It is based on off-the-shelf Wi-Fi technology. For economic reasons, Rural Wireless Mesh Networks (RWMN) are typically composed of only one gateway and a set of mesh routers (MRs) which aim to cover only areas of interest in the locality.

This article, originally published under IGI Global's copyright on January 1, 2020 will proceed with publication as an Open Access article starting on February 1, 2021 in the gold Open Access journal, International Journal of Operations Research and Information Systems (converted to gold Open Access January 1, 2021), and will be distributed under the terms of the Creative Commons Attribution License (http:// creativecommons.org/licenses/by/4.0/) which permits unrestricted use, distribution, and production in any medium, provided the author of the original work and original publication source are properly credited. 
The sole gateway usually connects the network to Internet via a last mile solution such as VSAT. The success of the planning of such networks depends on the determination of an optimal placement of mesh nodes which provides good coverage while requiring fewest nodes. The planning of wireless networks in rural regions is more coverage-driven than capacity-driven (Bernardi, Marina, Talamona, \& Rykovanov, 2011), which means we are more concerned by the area to cover than the capacity to provide. The aim during the planning is to minimize the overall cost of the architecture, while maximizing the coverage percentage of the area of interest. For realistic deployment scenarios, the problem of mesh node placement is a NP-hard combinatorial optimization problem which cannot be solved in polynomial time. This is why metaheuristics are usually required to optimize the planning.

Convergence time and robustness are important issues when it comes to developing or applying a stochastic optimisation technique. Simulated annealing (SA) is a probabilistic global optimisation technique used in large search spaces, which accepts some non-improving solutions in order to escape local optima. Since its introduction by Kirkpatrick et al. (1983), SA has been applied to a large variety of optimization scenarios, including the problem of node placement in Rural Wireless Mesh Networks (RWMN). Several years of experience using SA has led to the following general observations (Ingber, 1989):

- SA yields high-quality solutions, but may require large amounts of computation time;

- SA is especially advantageous in practical situations, where tailored algorithms are unavailable, due to its general applicability and its ease of implementation.

Several authors have attempted to reduce the convergence time for SA algorithms (Varanelli \& Cohoon, 1999; Yuping, Shouwei, \& Chunli, 2005). Other authors have recently tried to improve the solution quality of SA by providing hybrid approaches (Ezugwu, Adewumi, \& Frîncu, 2017; Chen, Chien, Chena, \& Chiena, 2011; Yannibelli \& Amandi, 2013; Jia, Ma, Wang, \& Liu, 2011). In this paper, we tackle the problem of improving the quality of solution and the robustness while minimising the convergence time of the SA approach in solving the problem of node placement in a Rural Wireless Mesh Network (RWMN). We consider the network model proposed in (Fendji, Thron, \& Nlong, 2015). In this model, a given area to cover is decomposed into elementary areas which are identified as "required" or "optional" in terms of coverage, and "possible" or "not possible" in terms of node placement. We consider also the presence of obstacles that can hinder the connectivity. The aim is therefore to determine the location of mesh routers which maximizes the coverage of area of interest while ensuring the connectivity. To reach this goal, a placement approach based on the calculation of the centre of mass $(\mathrm{CM})$ of area covered per router is proposed. This approach is later combined with the SA approach defined in (Fendji, Thron, \& Nlong, 2016) in order to obtain a hybrid method, Simulated Annealing based Centre of mass (SAC).

The rest of the paper is organized as follows: Section 2 briefly presents related work in WMN design. Section 3 defines the network model and formulates the placement problem. The SA optimization technique is presented in Section 4. Section 5 explains the three different approaches (SA, CM, SAC). Section 5 presents the experimental setup and compares the performance of the different approaches. This paper ends with a conclusion and future work.

\section{DESIGN OF WIRELESS MESH NETWORK}

Much research has been conducted in the design of WMNs: typically, the aim is to optimize some criteria related to the network configuration or operation. A good survey on the design of WMNs can be found in (Benyamina, Hafid, \& Gendreau, 2012), in which the design problem is classified according to the flexibility of the network topology: fixed (predefined) and unfixed (not-predefined). We discuss these two categories below. 


\subsection{Predefined Topology}

In predefined topologies, each mesh router is installed in a predefined location and cannot be moved elsewhere. The planning problem involves improving the way each node in the network sends and receives information. In particular, efficient use of transmission channels can reduce interference and increase the capacity of the network (Gupta \& Kumar, 2000). Some approaches include new MAC protocols (Garces \& Garcia-Luna-Aceves, 2000; Darties, Theoleyre, \& Duda, 2009); channel bonding technique (Xu, Yamamoto, \& Murata, 2008); cross layer design (Akyildiz \& Wang, 2008; Fu, Xiao, Deng, \& Zeng, 2014); network topology control design (Liu \& Liao, 2009; Marina, Das, \& Subramanian, 2010); network virtual backbone construction (F. Wang, Thai, \& Du, 2009); multichannel routing algorithms; channel assignment schemes (Das, Alazemi, Vijayakumar, \& Roy, 2005; Chaudhry, Hafez, Aboul-Magd, \& Mahmoud, 2010); and joining approaches. An interesting survey in joining approaches is found in (Pathak \& Dutta, 2011).

\subsection{Not-Predefined Topology}

In not-predefined topology, the planning problem aims to optimally place the nodes of the network: Internet gateway(s), mesh routers, or both.

\subsubsection{Internet Gateway(s) Placement}

The Internet gateway(s) placement problem has received much attention, since the gateway is the main mechanism for internetworking and is more expensive than simple mesh routers. The number of Internet gateway(s), their location and the number of hops in the network have a direct effect on the performance, the scalability, and the overall cost of the network. Planning approaches in the literature fall into two categories: cluster-based approaches, where the network is partitioned into subsets of nodes, each subset being served by one Internet gateway (Aoun, Boutaba, Iraqi, \& Kenward, 2006; Tang, 2009; Djohara Benyamina, Hafid, \& Gendreau, 2009; Jingzhi, Jianxiao, \& Zhifeng, 2009); and non-clustering approaches, which consider the network as a whole (Zhou, Wang, Manoj, \& Rao, 2010; Franklin \& Murthy, 2007; Xin \& Wang, 2009; Wenjia Wu, Junzhou Luo, \& Ming Yang, 2009).

Although there are different objectives in different gateway placement problems, they all take into account the placement of mesh routers.

\subsubsection{Mesh Router Placement}

In basic mesh router placement problems, the Internet gateways are taken as fixed, and mesh router locations are sought which optimise the objective(s) subject to given constraints. For example, in (Sen \& Raman, 2007), the authors take an existing landline node as the Internet gateway, and obtain a low-cost solution that connects neighbour villages. The costs related to the height of the antenna towers and the multi-hop topology are minimized subject to throughput, power and interference constraints. (Chen \& Chekuri, 2007) consider the planning of a robust and fault-tolerant urban WMN composed of mesh routers that use directional antennas. The aim in this planning problem is the maximisation of the deployment profit expressed in terms of amount of services provided, while maintaining the cost within the budget. In (Roh \& Lee, 2010), the authors consider a wireless network that consists of multiple fixed nodes and a single relay node that has controllable mobility. The objective was to maximise the weighted throughput of a node that achieves the lowest weighted throughput among all nodes.

\subsubsection{Planning From Scratch}

Unlike the basic placement problems described above, in most practical situations the mesh router placement and gateway placement problems are not taken independently, but rather are different aspects of the overall network planning problem. We refer to such cases as planning the network "from scratch". Planning a network from scratch consists not only in finding the 
optimal locations and node types in the network, but also in determining a judicious number of interfaces and channel assignment for each node while minimizing the cost and ensuring the connectivity, the required coverage and a minimum QoS. (Wang, Xie, Cai, \& Agrawal, 2007) provide an approach for node placement where the set of candidate positions is predetermined. The aim is to determine a minimum set of positions satisfying network coverage, connectivity and Internet traffic demand requirements. (Beljadid, Hafid, \& Gendreau, 2007) address a similar problem with the objective of minimising the overall cost of the network. (Amaldi, Capone, Cesana, Filippini, \& Malucelli, 2008) formulate the planning model of a WMN considering the coverage and topology planning for real size networks (60 nodes). They use a mixed integer linear programming model to solve their placement problem. (Wu, Luo, \& Yang, 2010) define the WMN placement problem as an integer linear program. They propose a method for determining mesh access point locations, Internet gateway selection and mesh router addition with the aim of minimising the cost of the network.

The earliest research in WMN planning used deterministic methods to solve the node placement problem, after formulating it as a single-objective linear programming problem. The main drawback of this formulation is the restriction of the network size, especially when the number of constraints is important. For realistically-sized networks, probability techniques are used, including: genetic algorithms (GA) (De Marco, 2009), (Xhafa, Sanchez, \& Barolli, 2010); Particle Swarm Optimisation (PSO) (Benyamina, Hafid, \& Gendreau, 2008), (Benyamina, Hallam, \& Hafid, 2008); Simulated Annealing (SA) (Xhafa, Barolli, Sanchez, \& Barolli, 2011); and ad hoc and neighbourhood search method (Xhafa, Sanchez, \& Barolli, 2009).

Some researchers provide a multi-objective formulation for the WMN planning problem. In (Xhafa et al., 2011), the problem is defined as a hierarchical multi-objective optimization problem in which a priority is defined between the two objectives of coverage and size of the giant component (connectivity). A non-hierarchical multi-objective formulation based on Pareto optimisation is provided in (Benyamina, Hafid, \& Gendreau, 2008a).

\subsection{WMN Planning in Rural Regions}

WMN planning in rural regions is usually framed as a mesh router placement problem, as in (Sen \& Raman, 2007) wherein different villages are connected from a landline node. WMN's in rural regions are usually composed of a set of mesh routers and a sole gateway that connects the network to the Internet via a last mile solution such like VSAT. This is the case of Macha network in Zambia (Backens, Mweemba, \& Van Stam, 2010).

The main constraint when planning a rural WMN is the cost of the solution. Since rural populations are typically sparsely distributed, planners may reduce cost without significantly affecting service by serving only areas presenting the highest potential usage. Users are typically not fixed, so the coverage constraint is more appropriately expressed as a specification of an area rather than a set of clients to serve. In this configuration, the planning problem resembles basic network planning with only the location of the Internet gateway (IGW) taken as known. Figure 1 illustrates a Rural Wireless Mesh Network with a sole gateway connected to Internet via VSAT. In contrast to urban regions where the planning of networks is typically capacitydriven, in rural regions the planning of networks is rather coverage-driven (Bernardi et al., 2011). Coverage-driven planning is especially appropriate in situations when most of the applications accessible through the network are text and audio-based rather than video-based: this is often the case for rural WMN's.

The work begun in (Fendji, Thron, \& Nlong, 2014) and extended in (Fendji et al., 2015) introduces a new placement problem of mesh routers in a rural region. Regions to be covered are decomposed into small elementary areas which may or may not require coverage or be available for node placement. A placement approach based on metropolis algorithm has therefore been used. 


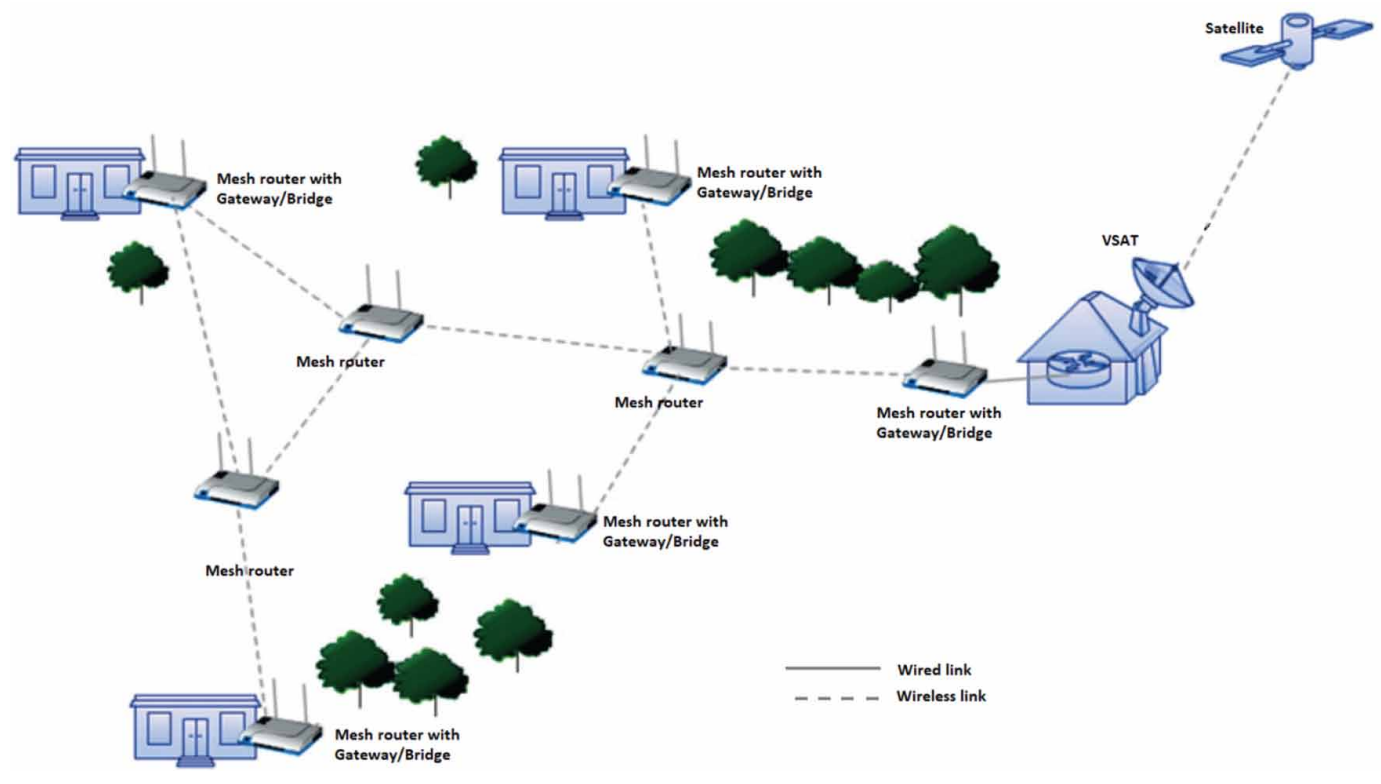

\section{FORMULATION OF THE PLACEMENT PROBLEM}

In previous works, the coverage constraint has been usually represented as a set of clients distributed in a region. Following (Fendji et al., 2015), here we consider instead the coverage constraint as an area to cover. In this placement problem, a given region is composed of areas of interest that should be covered. The coverage of a region is considered as optional when this region is not of interest. A given region comprised also prohibited areas where a node cannot be placed (lake, river, road, etc.), and a set of obstacles that could hinder the connectivity. The area to cover is modelled as a two-dimensional irregular form in a two-dimensional coordinate plane. We consider the smallest rectangle that can contain the irregular form, and divide this rectangle into small squares which are indexed by a pair of integers (x, y). These squares are called elementary areas (EA). Each EA can be of one or more types:

- EAI: Elementary Area of Interest

- OEA: Optional Elementary Area

- NEA: Non-line-of-sight (NLOS) Elementary Area

- PEA: Prohibited Elementary Area

Figure 2 illustrates the result of a decomposition of a region into a set of EA. When an EA is not an EAI, it is automatically an optional EA (OEA); that means an EA is either an EAI or an OEA. However, an EAI can be a NEA or a PEA. The network has a single Internet gateway, labelled as IGW.

In order to describe the situation mathematically, we can define several two-dimensional matrices to characterise each EA type: the matrix Cover indicates whether or not an EA requires coverage; Place indicates whether or not we can place a node in an EA; CoverDepth indicates the number of routers covering an EA; and Pathloss indicating whether or not an EA contains an obstacle. Specifically, matrix entries for position $(x, y)$. are defined by (1-4): 
Figure 2. Example of the decomposition of an area into EA



Cover $(\mathrm{x}, \mathrm{y})=\left\{\begin{array}{l}0 \rightarrow \text { coverage not required } \\ 1 \rightarrow \text { coverage required }\end{array}\right.$

Place $(x, y)=\left\{\begin{array}{l}0 \rightarrow \text { cannot place a node } \\ 1 \rightarrow \text { can place a node }\end{array}\right.$

CoverDepth $(\mathrm{x}, \mathrm{y})=\left\{\begin{array}{l}0 \rightarrow \text { no coverage } \\ n \rightarrow \text { covered by } n \text { routers }\end{array}\right.$

$\operatorname{Pathloss}(\mathrm{x}, \mathrm{y})=\left\{\begin{array}{l}0 \rightarrow \text { no obstruction } \\ p \rightarrow \text { attenuation factor }=p\end{array}\right.$

The following expressions are considered:

- The area to cover is denoted by $\mathcal{D} \subset \mathbb{N} \times \mathbb{N}$, since it is discretized into elementary elements;

- $M=\left\{R_{1}, R_{2}, \ldots, R_{n r}\right\}$ is the set of mesh routers;

- $\quad$ Each mesh router node has a unique integer identifier $j \in[1, n r]$;

- The sets of EA, EAI, and PEA are represented respectively by $\mathcal{A}, \mathcal{A} i$, and $\mathcal{A} p$;

- The number of EA, EAI, and PEA are given respectively by $n_{\text {ea }}=|\mathcal{A}|, n_{\text {eai }}=|\mathcal{A} i|$, and $n_{\text {pea }}=|\mathcal{A} p|$;

- The set of EA covered by $R_{j}, j \in[1, N] \cap \mathbb{N}$ is represented by $C \mathcal{A}_{j}$; 
- The set of EAI covered by $R_{j}, j \in[1, N] \cap \mathbb{N}$ is represented by $C \mathcal{A} i_{j}$;

- The set of EAI covered by $R_{j}, j \in[1, N] \cap \mathbb{N}$ alone is represented by $C \mathcal{A} i s_{j}$;

- The number of EAI covered by $R_{j}, j \in[1, N] \cap \mathbb{N}$ is represented by $\operatorname{Cov}_{j}=\left|C \mathcal{A} i_{j}\right|$;

- The number of EAI covered by $R_{j}, j \in[1, N] \cap \mathbb{N}$ along with at least one other MR is represented by $m \operatorname{Cov}_{j}=\left|C \mathcal{A} m_{j}\right|$;

- The number of EAI covered by $R_{j}, j \in[1, N] \cap \mathbb{N}$ alone is represented by $s \operatorname{Cov}_{j}=\left|C \mathcal{A} s_{j}\right|$;

- The centre of mass of the set of EAI covered by $R_{j}, j \in[1, N] \cap \mathbb{N}$ is represented by $C M_{j}$.

Let $p$ be an EA at position $(x, y)$. If $R_{j}$ is located in $p$, then the set of EAs covered by $R_{j}$ is given by (5):

$C \mathcal{A}_{j}=\left\{(\mathrm{a}, \mathrm{b}),(\mathrm{x}-\mathrm{a})^{2}+(\mathrm{y}-\mathrm{b})^{2}<\mathrm{r}_{j}^{2}\right\}$ with $\operatorname{Cov}_{j}=\left|C \mathcal{A}_{j}\right|$

For any $R_{j}, j \in[1, N] \cap \mathbb{N}$, the following equations hold:

$$
\begin{aligned}
& C \mathcal{A} s_{j} \cap C \mathcal{A} m_{j}=\varnothing \\
& C \mathcal{A} s_{j} \cup C \mathcal{A} m_{j}=C \mathcal{A} i_{j}
\end{aligned}
$$

\subsection{Assumptions}

To simplify the problem, we assume that the attenuation factor of any obstacle in the line of sight between two routers is high enough to prevent any wireless link between those routers. We also assume that all routers are equipped with an omnidirectional antenna and having the same transmission range. The transmission range is expressed in units of EA width, so for example $r=6$ means that the radius stretches across 6 EAs.

The mesh router placement problem in rural regions can be described as the determination of a minimum set of positions, which maximizes the coverage of areas of interest, while minimising the cost of the architecture. This cost can be minimised just by minimising the number of routers required to cover the region.

The basic placement problem of $n r$ routers in this area requires the evaluation of the number of combinations given by (8) for checking the optimal coverage:

$$
C_{|\mathcal{A}|-|\mathcal{A} p|}^{n_{r}}=\frac{(|\mathcal{A}|-|\mathcal{A} p|) !}{n_{r} !\left(|\mathcal{A}|-|\mathcal{A} p|-n_{r}\right) !}
$$

For a real-life scenario, we suppose $\mathcal{D}=50 \times 50, r=60,|\mathcal{A} p|=100$. We also suppose that half of the region is optional in terms of coverage. Thus, $|\mathcal{A} i|=\frac{50^{*} 50}{2}=1250$. Therefore, the number of combinations to examine is:

$$
C_{1150}^{60}=\frac{(1250-100) !}{60 !(1250-100-60) !}=\frac{1150 !}{60 ! 1090 !}
$$


This number increases exponentially with the size of $\mathcal{D}$ and $n r$. Since the number of combinations is too high, an exhaustive deterministic algorithm is not practically possible. Therefore, a meta-heuristic should be used.

\section{SIMULATED ANNEALING BASED CENTRE OF MASS (SAC)}

This section discusses the two basic algorithms that are combined to obtain the SAC algorithm for solving the problem of mesh nodes placement in RWMN.

\subsection{Simulated Annealing Algorithm}

The SA algorithm has been developed by (Kirkpatrick et al., 1983). It is inspired from the annealing process in metallurgy and material science, whereby a substance is heated to a specific temperature and then slowly cooled to reduce the defects and to obtain a strong crystalline structure reaching a minimal (optimal) energy level. The energy changes in the cooling process of a system are simulated by the SA algorithm until an equilibrium state (an optimum) is reached. The first development of an optimisation technique based on an annealing process dates back to Metropolis (Metropolis, Rosenbluth, \& Teller, 1953).

\subsubsection{Basic Algorithm}

The SA algorithm proceeds as follow: First, an initial solution is generated; several iterations are performed from this solution. A new random solution is generated at each iteration. If the solution improves the value of the objective function, it is directly accepted. Otherwise, this non-improving solution is accepted with a probability depending on the current temperature $T$ and the difference $\Delta E$.between the value of its objective function $f\left(s^{\prime}\right)$ and that of the previous solution $f(s)$. Since the temperature decreases progressively, the probability of accepting non-improving solutions also decreases. Usually, the probability in the SA algorithm follows the Boltzmann distribution given in (9) (Talbi, 2009) (Algorithm 1):

$$
P(\Delta E, T)=e^{\frac{-\Delta E}{T}}
$$

The basic version of the SA algorithm is given in Algorithm 1.

Algorithm 1. Simulated annealing for maximising problem

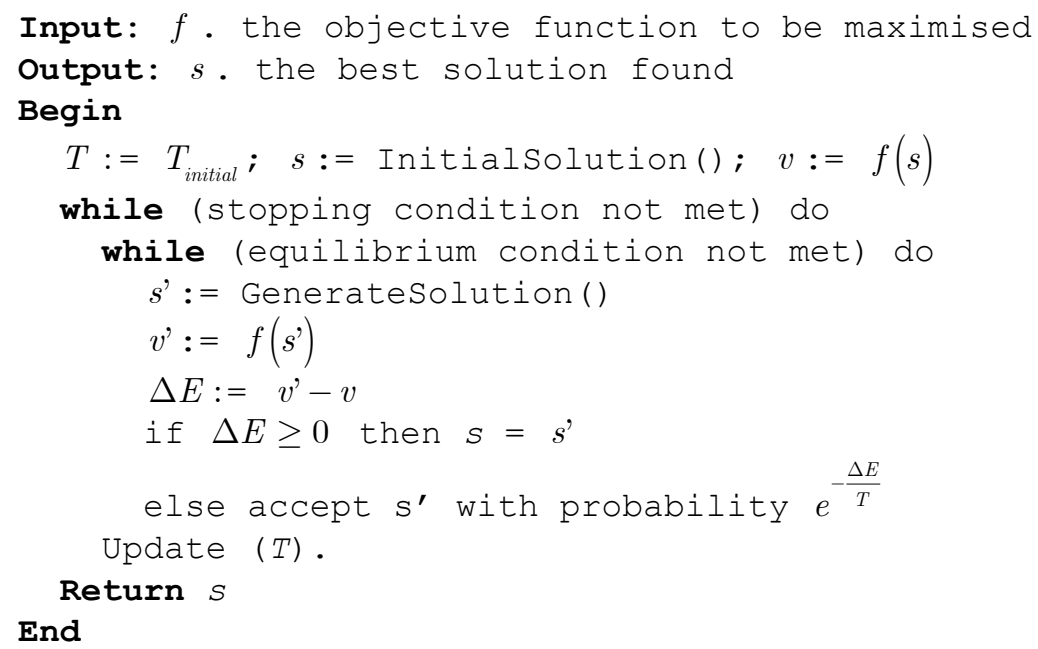




\subsubsection{Influencing Parameters}

Several parameters influence the efficiency of the SA algorithm, and should be optimized in order to minimize the computational time. The main parameters are: the initial temperature, the equilibrium state, the temperature update scheme, the acceptance probability, and the stopping condition (Talbi, 2009).

\subsubsection{Initial Temperature}

The initial temperature should not be too high in order to avoid the cooling process to be time consuming in the SA algorithm. But it should also not be too low to escape from local optima which may be near the initial solution.

\subsubsection{Equilibrium State}

An equilibrium state should be reached at each temperature; then the temperature is decreased. Two possible strategies may be used to indicate an equilibrium state: static or adaptive. For the static strategy, the number of moves to perform at each temperature is predefined: this strategy has drawbacks in that the number of iterations can be too long (increasing the running time of the algorithm) or too short (leading to suboptimal solutions). For the adaptive strategy, the number of moves is dynamic: for instance, a given number of moves without improvement of the solution can be considered as a sufficient condition to update the temperature.

\subsection{Temperature Update Scheme}

Temperature updates decrease in value as according to the following general scheme:

$$
\left(T_{i}>0\right) \wedge\left(T_{i}>T_{i+1}, \forall i \in \mathbb{N}\right) \wedge\left(\lim _{i \rightarrow \infty} T_{i}=0\right)
$$

The decrease of the temperature can be based on different schemes. The most common schemes of updates are: linear; geometric (e.g. Cauchy annealing); or logarithmic (e.g. Boltzmann annealing).

\subsubsection{Stopping Condition}

The most commonly-used stopping condition is to stop at a fixed temperature threshold. Another proposed stopping condition is to perform a number of a fixed number of temperature changes without improving the best solution found. If at a temperature $T_{i}$. the equilibrium state is reached without improvement of the solution, the algorithm can stop without necessarily reaching the final temperature.

\subsubsection{Simulated Annealing for RWMN}

The basic SA algorithm is a single solution-based metaheuristic (S-metaheuristic) that searches for the global optimum of a single objective function. It is usually used in a single-objective optimization problem. However, in our problem, both the extent of coverage and the number of routers is considered as objectives to be optimized. This means that the solutions that the algorithm will seek Pareto optimal solutions. We assume the number of routers to place to be unknown at the beginning in this placement approach.

The particularization of the SA algorithm aims to define suitable values of its parameters in order to enable the approach to be more efficient. We consider the particularization of SA approach found in (Fendji, Thron, \& Nlong, 2016). 


\subsubsection{Initial Solution}

The initial solution is obtained by placing routers randomly in the area to cover while ensuring that the centre of the router is located at an EA (x,y) for which Cover $(x, y)=1$, and Place $(x, y)=1$.

\subsubsection{Cooling Schedule}

The initial temperature $T=0.25$ has been determined empirically. We select a geometric update scheme as defined in (10) with $\alpha=0.5$. When the temperature is less than $T_{\text {min }}=0.01$, the cooling process stops:

$$
T_{i+1}=\alpha T_{i}
$$

\subsubsection{Move}

Only one router is moved at a time: both direction and distance are randomly selected based on a uniform distribution function. The movement from the current $\mathrm{EA}_{\mathrm{a}}=(x, y)$ to the new $\mathrm{EA}_{\mathrm{b}}=\left(x^{\prime}, y^{\prime}\right)$ is simulated if and only if Cover $\left(x^{\prime}, y^{\prime}\right)=1$ and $\operatorname{Place}\left(x^{\prime}, y^{\prime}\right)=1$. Initially great moves $(\mathrm{r}, \mathrm{r} / 2)$ are selected to allow a rapid convergence. The size of moves decreases with the temperature; when the temperature is close to $T_{\min }$, the size of moves is one EA.

\subsubsection{Fitness Function}

We count the number of EAIs that are covered to evaluate the fitness function. This is done by (11) after the initialization:

$$
\mathrm{f}=\sum \operatorname{sign}\left(\text { CoverDepth. }{ }^{*} \text { Cover }\right)
$$

Since we move only one router at the same time, we consider also only the EAs which are affected by the motion.

\subsubsection{Acceptance Criterion}

When moving a selected router $R_{j}$ from an $\mathrm{EA}_{\mathrm{a}}=(x, y)$ to an $\mathrm{EA}_{\mathrm{b}}=\left(x^{\prime}, y^{\prime}\right)$ if $C \mathcal{A}$ is increases $_{j}$ (that means $\Delta E$ is positive), then the coverage is directly accepted. But if $C \mathcal{A} i s_{j}$ decreases (that means $\Delta E$ is negative), the change is accepted with a certain probability following the Boltzmann distribution given in (9).

\subsubsection{Equilibrium State and Stopping Condition}

The equilibrium state for a given temperature is presumed to be reached if after a number (Stop) of moves no solution has been accepted. The stopping condition depends on Imp and on $T_{\min }$. At each temperature $T_{i}$, Imp indicates whether the solution has improved. When the equilibrium state at a temperature $T_{i}$ is reached, before decreasing the temperature we check whether the solution has improved. In case of an improvement, we decrease the temperature and move to the next iteration. But if there is no improvement or the temperature is less than $T_{\min }$, we stop the search process and suppose having reached an optimum. 


\subsection{Centre of Mass of Single Coverage (CM)}

\subsubsection{Algorithm}

The idea behind the approach of the centre of mass of single coverage is to reduce the area covered by multiple routers by moving routers in high-coverage regions to the centre of mass of areas that are not yet covered. This approach is motivated by the fact that by moving routers to the centre of mass of their single coverage, new non-covered EAI can be reached in a relative short number of moves. Since moves effectively reduce multiple coverage, the coverage improves more rapidly than with the SA approach. In fact, the SA approach sometimes moves routers to areas that are already covered. The CM algorithm is given in Algorithm 2.

Algorithm 2. Centre of mass of single coverage

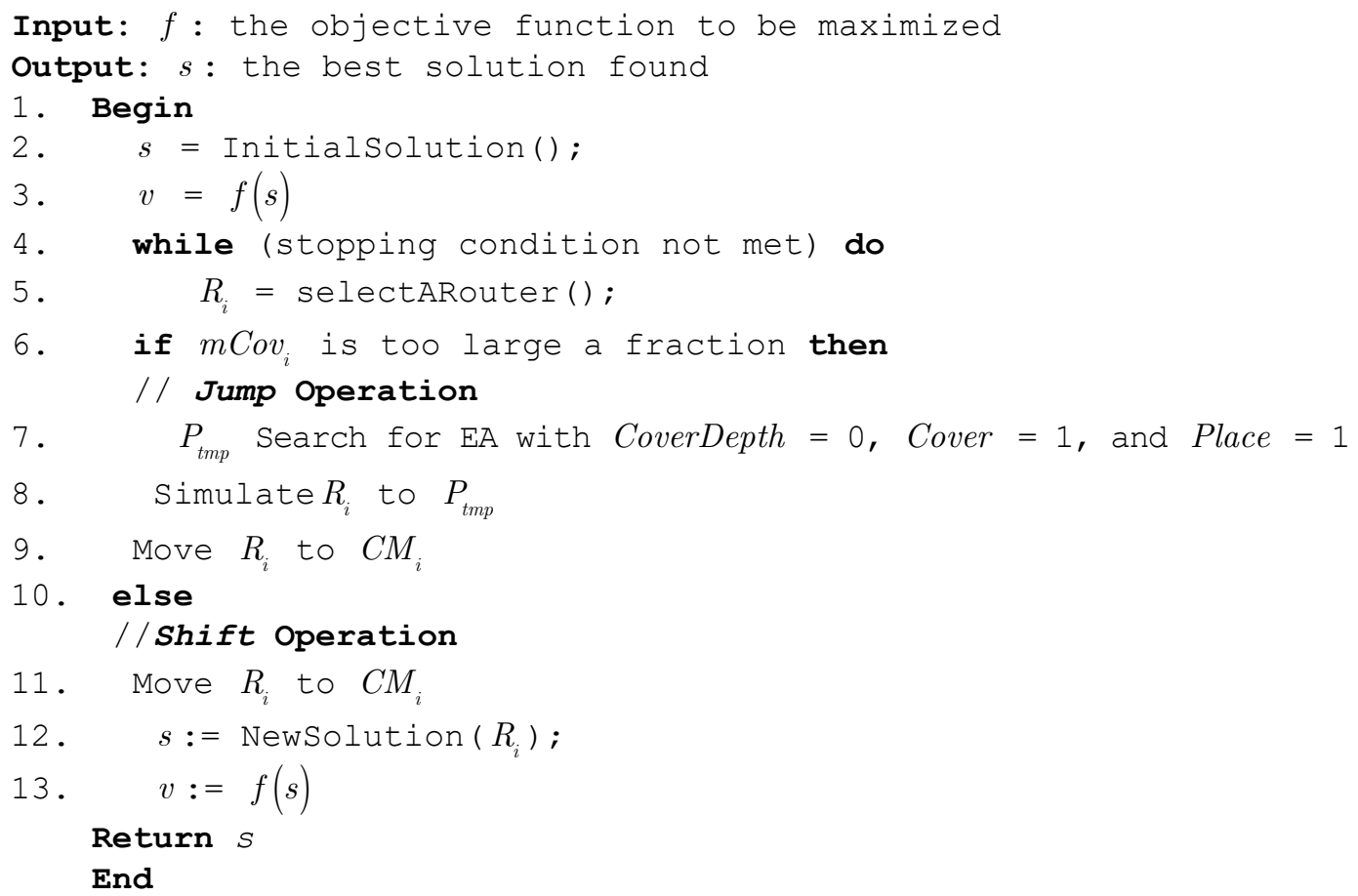

\subsubsection{Algorithm Explanation}

- Initial Solution: The initial solution is obtained, as in SA algorithm, by placing routers randomly in the area to cover while ensuring that $\operatorname{Cover}(x, y)=1$, and Place $(x, y)=1$;

- Moves based on single or multiple coverage: To improve the initial solution, a router $R_{j}$ is moved at the same time. Two types of move are defined depending on $m \operatorname{Cov}_{j}$ and $s \operatorname{Cov}_{j}:$ Shift operation and Jump operation;

- Shift operation: Consists into moving $R_{j}$ to $C M_{j}$. That means $R_{j}$ is moved to the Centre of Mass of its single coverage $C \mathcal{A} i s_{j}$. An example of Shi operation is illustrated in Figure 3c;

- Jump operation: Consists into first randomly selecting an $\mathrm{EA}=(x, y)$ with $\operatorname{CoverDepth}(x, y)=0$, Cover $(x, y)=1$, and Place $(x, y)=1$; then relocating $R_{j}$ to $C M_{j}$, which is the Centre of Mass of the single coverage of the new selected EA. An example of Jump operation is illustrated in Figure 3b. 
Figure 3. (a) Configuration at a time $t$; (b) Configuration at a time $t+1$ after a Jump operation if black router is selected; (c) Configuration at a time $t+1$ after a Shift operation if Red router is selected

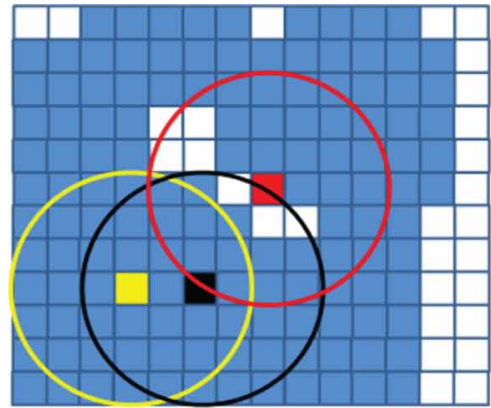

(a)

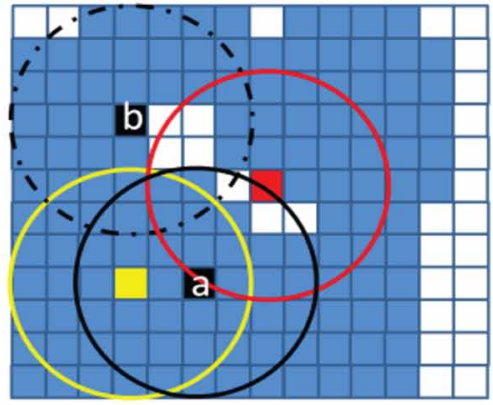

(b)

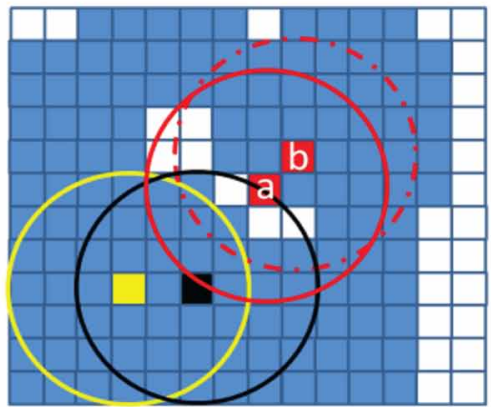

(c)

Shift and Jump operations are exclusive and selected depending on condition (12). $\operatorname{rand}(x)$ is a function following a uniform distribution. We can remark that when $s \operatorname{Cov}_{j}$ is too great compared to $m \operatorname{Cov}_{j}$, expression in (12) has a great probability to be not satisfied. If it is the case, the shift operation is applied on $R_{j}$ reducing eventually its multiple coverage. Otherwise the Jump operation is applied:

$$
\left(\operatorname{sov}_{j}+m \operatorname{Cov}_{j}\right)^{2} * \operatorname{rand}(\mathrm{x})<\left(m \operatorname{Cov}_{j}\right)^{2}
$$

- Fitness Function (lines 3 and 13): The evaluation of fitness function consists, as it is the case in the SA algorithm, to count the number of covered EAI. This is done by (11) after the initialization;

- New Solution (line 12): It is obtained by keeping other routers in their previous positions and considering the new position of router $i$;

- Stopping Condition: If the value of the fitness function does not improve after a certain number of iteration TTS (Time to search), we suppose therefore having reached an optimum. The value of TTS has a direct incidence on the convergence time of the algorithm. A small value leads automatically to a rapid convergence, while a great value will extend the running time of the algorithm.

\subsection{SAC Framework for Solving RWMN Problem}

SAC approach is a hybrid of Centre of Mass and Simulated Annealing. It is composed of two main phases: A Speedup phase based on Centre of Mass approach, and a Refinement phase 
based on Simulated Annealing. Flowchart is given in Figure 4. Best is an archive which keeps the best solution for each number of routers. nRun is the number of times the SAC algorithm will be executed.

The number of routers is unknown at the beginning and the form of the region is irregular. Since routers should overlap to ensure the connectivity, we start with a great number of routers, and then we reduce this number while trying to maintain a large coverage percentage of the area of interest. A lower bound to the number of routers to cover of the area of interest when all routers have the same transmission range is given by (13). A too great number of routers at the beginning is not efficient since the reduction of the number of routers will last longer. The initial number of routers was chosen between 1.5-2 times the lower bound, as in (14):

$$
\begin{aligned}
& n r_{\text {min }}=\sum \operatorname{Cover}(\mathrm{x}, \mathrm{y}) /\left(\mathrm{r}^{2 *} 3.14\right) \\
& \frac{3}{2} n r_{\text {min }} \leq n r_{\text {init }} \leq 2 n r_{\text {min }}
\end{aligned}
$$

\subsection{Initial Solution}

Routers are placed randomly in the region during the initialization phase of the SAC algorithm, but only in areas of interest. We randomly select an EA for each router. We check if Cover $(\mathrm{EA})=$ 1 , and $\operatorname{Place}(\mathrm{EA})=1$, and, if a router is not already set at this location then the current router can

Figure 4. Flowchart of the SAC approach for solving mesh nodes placement in RWMN

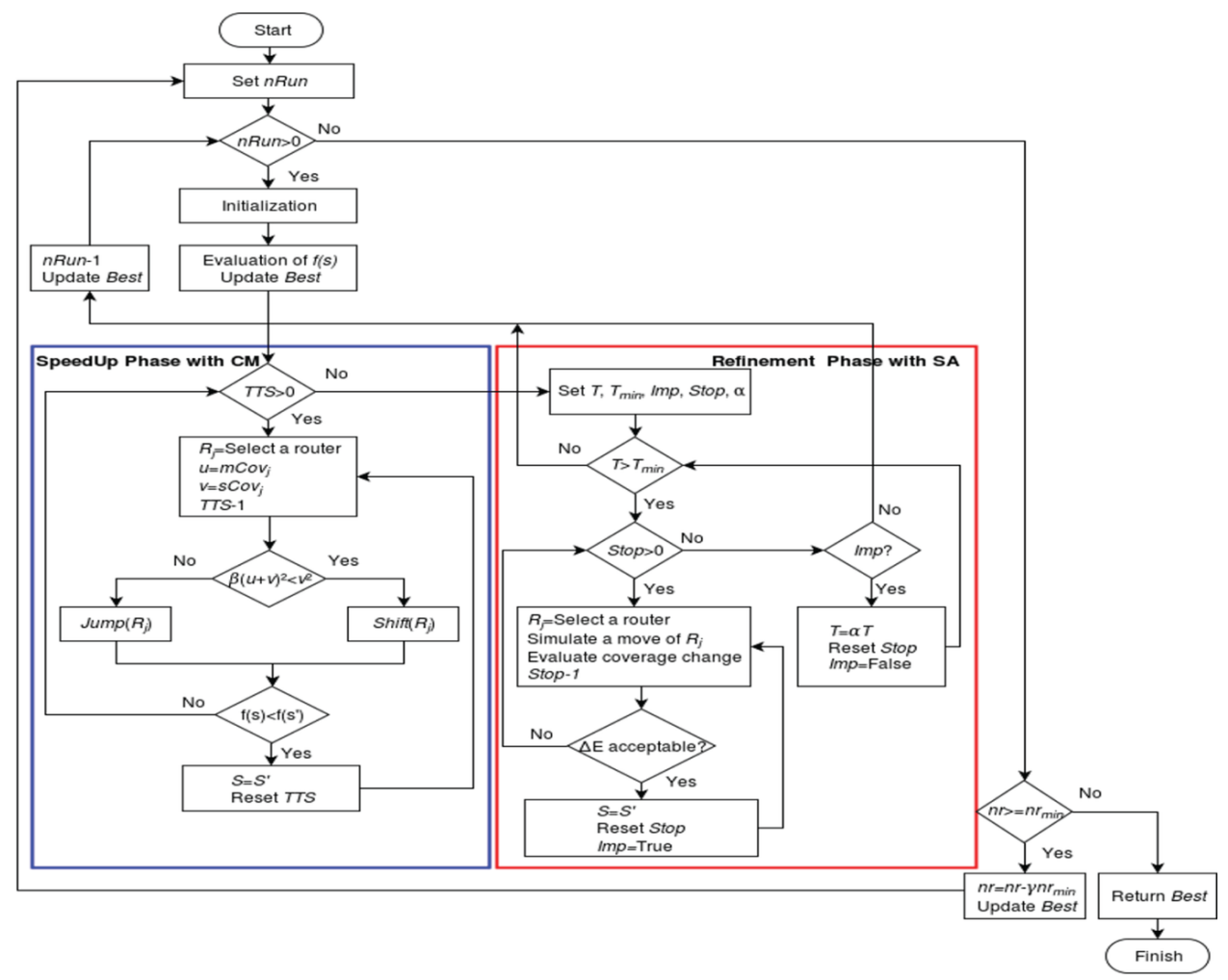


be placed there. Otherwise, we continue by selecting another EA. The initialization ends when all routers are placed.

\subsection{SpeedUp Phase With CM}

The aim of this phase coming right after the initialization is to achieve a rapid exploration of the search space. This stage consists of CM algorithm. TTS is set at the beginning. A router is randomly chosen and the suitable operation between Jump and Shift is performed. If there is no improvement of the current solution, TTS decreases; otherwise it is reset. This phase stops when TTS reaches 0 .

\subsection{Refinement Phase With SA}

This stage aims to improve the intermediate solution provided by the Speedup phase. This is achieved by the SA algorithm which make and exploitation of the search space in the neighbourhood of the intermediate solution.

\subsection{Reduction of the Number of Routers and Stopping Condition}

At the beginning $n r_{\text {init }}$ routers are used. The SAC algorithm is running $n R u n$ times for each number of routers, and the best solution is kept in Best. After completing the runs for $n r$ routers, the number of routers is reduced according to (15). The algorithm stops when $n r$ becomes less than $n r_{m i n}$ :

$$
n r=n r-\gamma \mathrm{nr}_{\min }
$$

\section{PERFORMANCE EVALUATION}

\subsection{Performance Metrics}

Different metrics are considered for the evaluation of performance of the presented algorithms.

\subsubsection{Coverage Percentage of Area of Interest}

The first metric is the coverage percentage of the area of interest. It is determined by the fitness function defined in (11). The closer this percentage is to 100 percent, the better is the quality of the solution. Since we reduce the number of routers progressively, we compare the algorithms' performance for each given number of routers; best case, mean and median coverage percentages are recorded after nRun runs of the algorithm.

\subsubsection{Robustness}

An important characteristic of stochastic optimisation approaches is the robustness, defined as the ability to provide similar results after repeated. The robustness reflects the degree of consistency (or reproducibility) in values obtained by the stochastic approach. We compute two measures of robustness: the standard deviation and interquartile range (IQR) of coverage percentages of the area of interest for all the runs with the same number of routers.

\subsubsection{Convergence Time}

The convergence time is another important consideration, particularly in online optimisation where the time to reach a solution is a practical constraint. In general, better quality solutions are obtained at the expenses of longer running times. We evaluate the convergence time by computing the best, the mean, and the median values of all runs: and we evaluate the dispersion by calculating the standard deviation and the IQR. 


\subsection{Simulations and Discussion}

\subsubsection{Network Instances}

Due to the lack of a suitable testbed, we created our own for this study. The testbed was designed to mimic real-world scenarios. We randomly generated 24 different regions with irregular forms, with areas of interest and prohibited areas. Four typical regions are presented in Figure 5. Since algorithms may perform differently in both runtime and solution quality on small and large problems, we consider four different grid sizes: $50 \times 50,100 \times 100,150 \times 150$, and $200 \times 200$. If size(EA) is taken to be $20 \mathrm{~m}$, then the selected grid sizes correspond to areas between $1 \mathrm{~km}^{2}$ and $16 \mathrm{~km}^{2}$. The completed testbed is available online (Fendji, 2017).

\subsubsection{Algorithm Parameters and Simulation Environment}

The algorithms parameters are given in Table 1. The transmission range $r$ is set to 6 . If size(EA) $=20 \mathrm{~m}$, the radius will be $6 \times 20 \mathrm{~m}=120 \mathrm{~m}$. This is realistic since $802.11 \mathrm{n}$ routers have a theoretical

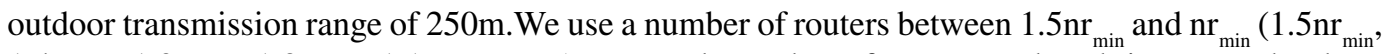
$1.4 \mathrm{nr}_{\min }, 1.3 \mathrm{nr}_{\min }, 1.2 \mathrm{nr}_{\min }, 1.1 \mathrm{nr}_{\min }, \mathrm{nr}_{\min }$ ). For each number of routers and each instance, the three algorithms are run 20 times. Simulations of all algorithms are conducted using SCILAB 5.5 on a 2.8 GHz CPU Desktop with 4GB RAM.

Figure 5. Four Instances in a set of 24 with irregular forms: (a) Instance 1 with $\mathcal{D}=50 \times 50$, (b) Instance 2 with $\mathcal{D}=100 \times 100$, (c) Instance 3 with $\mathcal{D}=150 \times 150$, (d) Instance 4 with $\mathcal{D}=200 \times 200$

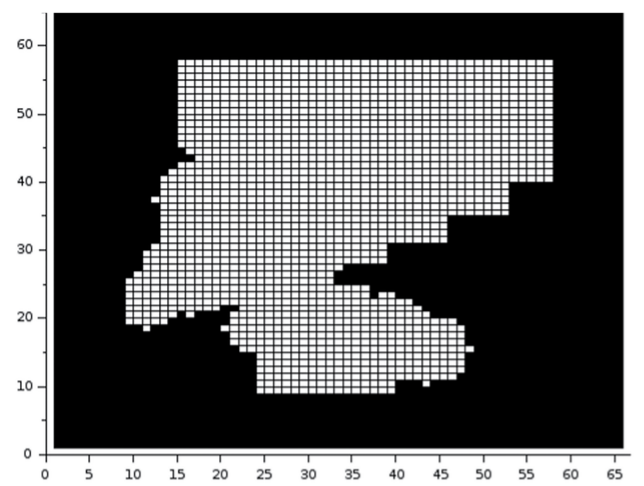

(a)

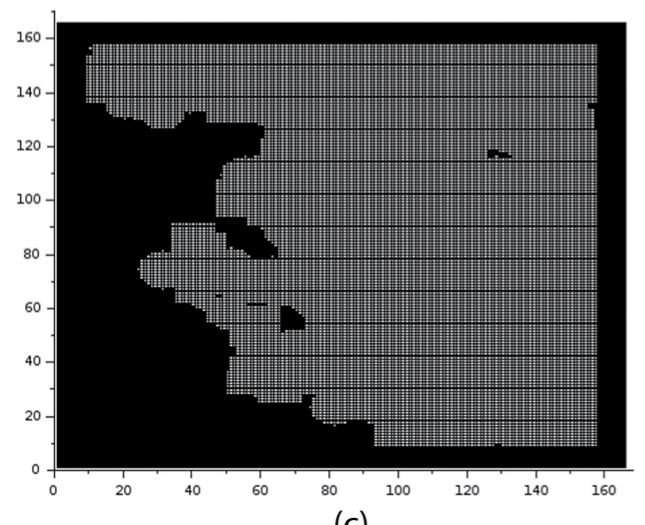

(c)



(b)



(d) 
Table 1. Algorithm parameters

\begin{tabular}{|l|l|}
\hline \multicolumn{1}{|c|}{ Parameter } & \\
\hline $\mathrm{TTS}$ & 1000 \\
\hline $\mathrm{T}_{\text {init }}$ & 0.25 \\
\hline$\alpha$ & 0.5 \\
\hline $\mathrm{T}_{\min }$ & 0.01 \\
\hline Stop & 1000 \\
\hline $\mathrm{nRun}$ & 20 \\
\hline $\mathrm{r}$ & 6 \\
\hline$\beta$ & {$[0,1]$} \\
\hline$\gamma$ & 0.1 \\
\hline $\mathrm{nr}_{\text {init }}$ & $1.5 \mathrm{nr}_{\text {min }}$ \\
\hline
\end{tabular}

\subsubsection{Discussion of Results}

Table 2 presents the mean, the median, and the best coverage percentages of the algorithms. The table shows that $\mathrm{CM}$ provides the worst results in terms of coverage percentage of AI, irrespective of the number of routers and the size of instances. SAC and SA are consistently very close by all three measures, and both SA and SAC provide coverage over 99 percent when $\mathrm{Nr} \geq 1.3 \mathrm{nr}_{\min }$. However, SAC consistently provides better results than SA in all metrics for large grids: at grid size $200 \times 200$, the mean and median coverage improvements over all metrics and router numbers is 0.4 percent. Figure 6 presents standard box plots of coverage percentages for the three different algorithms for values of $50 \times 50$ and $200 \times 200$ grids.

Figure 7 shows coverage percentages as a function of number of routers $(\mathrm{Nr})$ for the three algorithms for different grid sizes. As expected, the coverage percentages consistently decrease as

Table 2. Comparison of mean, median, and best coverage percentages of algorithms

\begin{tabular}{|c|c|c|c|c|c|c|c|c|c|c|c|c|c|}
\hline \multirow{2}{*}{$\begin{array}{c}\text { Number } \\
\text { of } \\
\text { Routers }\end{array}$} & & \multicolumn{3}{|c|}{$50 \times 50$} & \multicolumn{3}{|c|}{$100 \times 100$} & \multicolumn{3}{|c|}{$150 \times 150$} & \multicolumn{3}{|c|}{$200 \times 200$} \\
\hline & & CM & SA & SAC & CM & SA & SAC & CM & SA & SAC & CM & SA & SAC \\
\hline \multirow{3}{*}{$1,5 \mathrm{nr}_{\min }$} & Mean & 97.60 & 99.86 & 99.95 & 96.26 & 99.78 & 99.93 & 95.96 & 99.53 & 99.93 & 95.98 & 99.60 & 99.90 \\
\hline & Median & 97.84 & 100.00 & 100.00 & 96.48 & 99.84 & 99.96 & 96.16 & 99.74 & 99.94 & 96.16 & 99.64 & 99.93 \\
\hline & Best & 99.31 & 100.00 & 100.00 & 97.42 & 100.00 & 100.00 & 96.81 & 99.99 & 100.00 & 96.81 & 99.91 & 99.98 \\
\hline \multirow{3}{*}{$1,4 \mathrm{nr}_{\min }$} & Mean & 95.22 & 99.55 & 99.47 & 94.77 & 99.62 & 99.79 & 94.17 & 99.28 & 99.81 & 94.30 & 99.09 & 99.74 \\
\hline & Median & 95.85 & 99.94 & 99.84 & 94.77 & 99.71 & 99.85 & 94.46 & 99.54 & 99.84 & 94.46 & 99.31 & 99.79 \\
\hline & Best & 97.91 & 100.00 & 100.00 & 96.38 & 99.96 & 99.97 & 95.40 & 99.86 & 99.95 & 95.40 & 99.85 & 99.94 \\
\hline \multirow{3}{*}{$1,3 \mathrm{nr}_{\min }$} & Mean & 93.43 & 98.94 & 99.12 & 92.56 & 99.20 & 99.31 & 91.89 & 98.69 & 99.31 & 91.99 & 98.45 & 99.16 \\
\hline & Median & 94.53 & 99.53 & 99.50 & 92.65 & 99.18 & 99.36 & 92.33 & 99.01 & 99.39 & 92.33 & 98.86 & 99.35 \\
\hline & Best & 96.81 & 100.00 & 100.00 & 94.23 & 99.73 & 99.80 & 93.31 & 99.61 & 99.77 & 93.31 & 99.48 & 99.60 \\
\hline \multirow{3}{*}{$1,2 \mathrm{nr}_{\min }$} & Mean & 90.12 & 96.83 & 96.58 & 89.91 & 97.84 & 97.79 & 89.25 & 97.39 & 98.02 & 89.36 & 97.18 & 97.97 \\
\hline & Median & 91.70 & 98.34 & 97.96 & 89.97 & 97.97 & 97.95 & 89.62 & 97.81 & 98.11 & 89.62 & 97.58 & 98.13 \\
\hline & Best & 95.02 & 99.71 & 99.65 & 91.48 & 98.82 & 98.85 & 90.95 & 98.83 & 98.91 & 90.95 & 98.35 & 98.68 \\
\hline \multirow{3}{*}{$1,1 \mathrm{nr}_{\min }$} & Mean & 87.47 & 94.85 & 94.74 & 86.14 & 95.30 & 95.23 & 85.79 & 95.32 & 95.38 & 86.01 & 94.76 & 95.71 \\
\hline & Median & 88.23 & 96.07 & 95.63 & 86.27 & 95.38 & 95.33 & 86.20 & 95.49 & 95.68 & 86.20 & 95.16 & 95.75 \\
\hline & Best & 92.09 & 97.45 & 97.34 & 87.73 & 96.69 & 96.46 & 87.41 & 96.83 & 96.51 & 87.41 & 96.18 & 96.38 \\
\hline \multirow{3}{*}{$\mathrm{nr}_{\min }$} & Mean & 81.44 & 89.59 & 89.64 & 81.58 & 91.21 & 91.08 & 81.41 & 91.34 & 91.55 & 81.92 & 90.99 & 91.57 \\
\hline & Median & 82.85 & 91.02 & 90.57 & 81.75 & 91.46 & 91.13 & 81.72 & 91.63 & 91.75 & 81.72 & 91.34 & 91.67 \\
\hline & Best & 86.67 & 93.05 & 92.87 & 83.52 & 92.65 & 92.65 & 83.26 & 92.89 & 92.74 & 83.26 & 92.62 & 92.23 \\
\hline
\end{tabular}


Figure 6. Average coverage percentage provided by the different algorithms: (a) Instance 1 with $\mathcal{D}=50 \times 50$; (b) Instance 4 with $\mathcal{D}=200 \times 200$

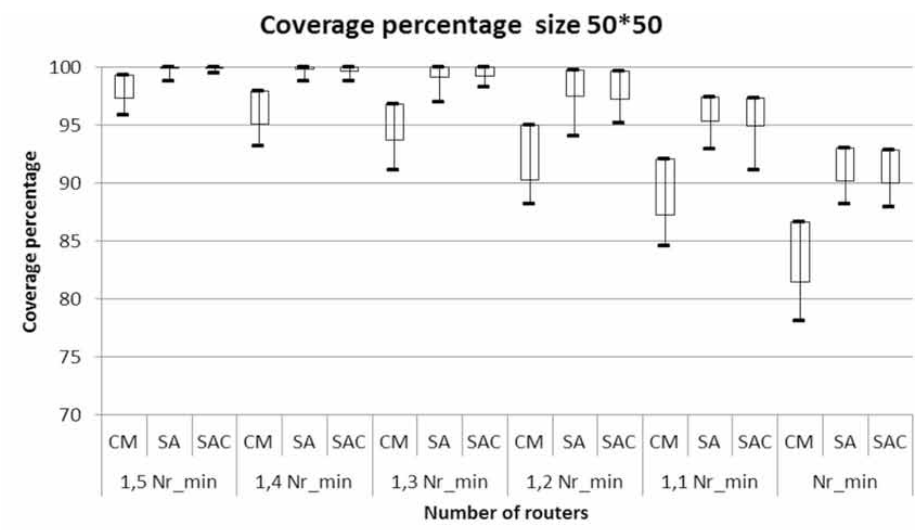

(a)



(b)

$N r$ decreases. When $N r=\mathrm{nr}_{\text {min }}$, SA and SAC achieve over 90 percent coverage independent of grid size: when $N r=1.3 \mathrm{nr}_{\text {min }}$, this increases to 98 percent. Larger grid sizes are associated with higher coverage percentages, and the difference increases as $\mathrm{Nr}$ decreases. The largest difference achieved between $50 \times 50$ and $200 \times 200$ coverage rates was 2 percent, at $N r=\mathrm{nr}_{\min }$ with SAC.

Table 3 presents the standard deviation and IQR of coverage percentages provided by the three algorithms. As far as relative performance of the three algorithms, the situation is similar to coverage percentage metrics. CM is consistently worst, and for the smallest grid sizes SA and SAC are comparable. But for larger grid sizes, the robustness measures for SAC are about 2-7 times smaller than those for SA: the improvement tends to be larger for larger router numbers. Apart from this, we may note a general tendency towards greater robustness for larger grid sizes, and larger router numbers.

Two-sided p-values have been computed to test the statistical significance when comparing SAC to other approaches. The p-value is computed for each number of routers by computing means and standard deviations for the twenty runs of each of the six instances for a given grid size (120 runs total) for the two algorithms being compared. When comparing CM with SAC, the p-value was always less than 0.00001 , indicating that SAC improvements over CM are highly statistically significant. The $\mathrm{p}$-values for SAC and SA are presented in Table 4. From this Table, SA provides a better coverage than SAC only with 50x50 grid and when the number of routers is less than $1.3 \mathrm{nr}_{\text {min }}$. For the smallest 
Figure 7. Average coverage percentage provided by the different algorithms



Table 3. Comparison of standard deviation and IQR of coverage percentage for three algorithms

\begin{tabular}{|c|c|c|c|c|c|c|c|c|c|c|c|c|c|}
\hline \multirow{2}{*}{$\begin{array}{c}\text { Number } \\
\text { of } \\
\text { Routers }\end{array}$} & & \multicolumn{3}{|c|}{$50 \times 50$} & \multicolumn{3}{|c|}{$100 \times 100$} & \multicolumn{3}{|c|}{$150 \times 150$} & \multicolumn{3}{|c|}{$200 \times 200$} \\
\hline & & $\mathbf{C M}$ & SA & SAC & CM & SA & SAC & CM & SA & SAC & $\mathbf{C M}$ & SA & SAC \\
\hline \multirow{2}{*}{$1,5 \mathrm{nr}_{\min }$} & Dev & 0.69 & 0.16 & 0.07 & 0.49 & 0.28 & 0.06 & 0.35 & 0.32 & 0.05 & 0.26 & 0.30 & 0.04 \\
\hline & IQR & 0.92 & 0.05 & 0.00 & 0.63 & 0.23 & 0.06 & 0.50 & 0.29 & 0.05 & 0.50 & 0.34 & 0.04 \\
\hline \multirow{2}{*}{$1,4 \mathrm{nr}_{\min }$} & Dev & 1.04 & 0.24 & 0.26 & 0.52 & 0.41 & 0.12 & 0.42 & 0.35 & 0.08 & 0.29 & 0.42 & 0.08 \\
\hline & IQR & 1.41 & 0.18 & 0.28 & 0.55 & 0.37 & 0.16 & 0.56 & 0.34 & 0.11 & 0.56 & 0.58 & 0.11 \\
\hline \multirow{2}{*}{$1,3 \mathrm{nr}_{\min }$} & Dev & 1.12 & 0.51 & 0.37 & 0.56 & 0.53 & 0.25 & 0.46 & 0.49 & 0.17 & 0.32 & 0.52 & 0.15 \\
\hline & IQR & 1.57 & 0.62 & 0.53 & 0.75 & 0.60 & 0.34 & 0.58 & 0.61 & 0.21 & 0.58 & 0.56 & 0.17 \\
\hline \multirow{2}{*}{$1,2 \mathrm{nr}_{\min }$} & Dev & 1.54 & 1.03 & 0.95 & 0.70 & 0.94 & 0.41 & 0.49 & 0.62 & 0.28 & 0.38 & 0.48 & 0.21 \\
\hline & IQR & 2.38 & 1.42 & 1.47 & 0.96 & 0.86 & 0.54 & 0.67 & 0.60 & 0.37 & 0.67 & 0.52 & 0.27 \\
\hline \multirow{2}{*}{$1,1 \mathrm{nr}_{\min }$} & Dev & 1.51 & 0.97 & 1.03 & 0.77 & 0.78 & 0.46 & 0.57 & 0.67 & 0.39 & 0.43 & 0.62 & 0.26 \\
\hline & IQR & 2.05 & 1.21 & 1.38 & 1.03 & 0.86 & 0.65 & 0.72 & 0.72 & 0.60 & 0.72 & 0.75 & 0.42 \\
\hline \multirow{2}{*}{$\mathrm{nr}_{\min }$} & Dev & 1.67 & 1.14 & 1.05 & 0.74 & 0.86 & 0.60 & 0.59 & 0.73 & 0.38 & 0.51 & 0.58 & 0.27 \\
\hline & IQR & 2.40 & 1.34 & 1.32 & 0.99 & 1.08 & 0.76 & 0.76 & 0.91 & 0.54 & 0.76 & 0.75 & 0.38 \\
\hline
\end{tabular}

number of routers $\left(\mathrm{nr}_{\min }\right.$ and $1.1 \mathrm{nr}_{\text {min }}$ ) in 100x100 grid, the superiority of one approach over the second one cannot be proved. However, for larger grid sizes, the superiority of SAC over SA in terms of coverage is confident since $\mathrm{p}$ is always less than 0.05 .

Table 5 presents the mean and median CPU times required by the different algorithms. CM approach provides the shortest running time, regardless the number of routers and the size of instances. However, this is clearly tied to the relatively poor coverage performance of $\mathrm{CM}$ - the CM algorithm explores less of the solution space, so the running time is less but better solutions are missed. SA provides the worst CPU time, ranging from about three times the CPU time used by the CM approach for small instances, up to twelve times for larger instances. Compared to SA, SAC shows 40-60 percent 
Table 4. Two-sided p-values when comparing mean coverage percentages of SAC and SA. Numbers in italics correspond to instances where the SA mean coverage percentage is higher than SAC, while numbers in regular case indicate instances where SAC mean percentage coverage was higher than for SA. Significance at $p<0.05$ is indicated in bold.

\begin{tabular}{|l|l|l|l|l|}
\hline Number of Routers & \multicolumn{1}{|c|}{$\mathbf{5 0 \times 5 0}$} & \multicolumn{1}{|c|}{$\mathbf{1 0 0 \times 1 0 0}$} & \multicolumn{1}{|c|}{$\mathbf{1 5 0 \times 1 5 0}$} & \multicolumn{1}{c|}{$\mathbf{2 0 0 \times 2 0 0}$} \\
\hline $1,5 \mathrm{nr}_{\min }$ & $\mathbf{0 . 0 1 3 6 4}$ & $\mathbf{0 . 0 0 0 0 1}$ & $\mathbf{0 . 0 0 0 0 1}$ & $\mathbf{0 . 0 0 0 0 1}$ \\
\hline $1,4 \mathrm{nr}_{\min }$ & $\mathbf{0 . 0 0 7 0 4 7}$ & $\mathbf{0 . 0 0 0 0 1}$ & $\mathbf{0 . 0 0 0 0 1}$ & $\mathbf{0 . 0 0 0 0 1}$ \\
\hline $1,3 \mathrm{nr}_{\min }$ & 0.393277 & $\mathbf{0 . 0 0 0 0 1}$ & $\mathbf{0 . 0 0 0 0 1}$ & $\mathbf{0 . 0 0 0 0 1}$ \\
\hline $1,2 \mathrm{nr}_{\min }$ & 0.022995 & $\mathbf{0 . 0 1 2 8 9 9}$ & $\mathbf{0 . 0 0 0 0 1}$ & $\mathbf{0 . 0 0 0 0 1}$ \\
\hline $1,1 \mathrm{nr}_{\min }$ & 0.004611 & 0.12259 & $\mathbf{0 . 0 0 0 2 1 5}$ & $\mathbf{0 . 0 0 0 0 1}$ \\
\hline $\mathrm{nr}_{\text {min }}$ & 0.024758 & 0.213914 & $\mathbf{0 . 0 0 0 2 0 4}$ & $\mathbf{0 . 0 0 0 0 1}$ \\
\hline
\end{tabular}

Table 5. Comparison of mean and median CPU times of algorithms

\begin{tabular}{|c|c|c|c|c|c|c|c|c|c|c|c|c|c|}
\hline \multirow{2}{*}{ Number of Routers } & & \multicolumn{3}{|c|}{$50 \times 50$} & \multicolumn{3}{|c|}{$100 \times 100$} & \multicolumn{3}{|c|}{$150 \times 150$} & \multicolumn{3}{|c|}{$200 \times 200$} \\
\hline & & CM & SA & SAC & $\mathbf{C M}$ & SA & SAC & $\mathbf{C M}$ & SA & SAC & CM & SA & SAC \\
\hline \multirow{2}{*}{$1,5 \mathrm{nr}_{\min }$} & Mean & 9.91 & 28.53 & 13.41 & 11.55 & 63.58 & 24.55 & 12.80 & 107.82 & 45.83 & 14.97 & 169.20 & 69.61 \\
\hline & Median & 9.23 & 28.83 & 13.32 & 11.07 & 63.19 & 26.30 & 12.39 & 115.28 & 46.40 & 14.25 & 170.62 & 70.97 \\
\hline \multirow{2}{*}{$1,4 \mathrm{nr}_{\min }$} & Mean & 8.50 & 29.88 & 14.24 & 9.94 & 61.82 & 29.80 & 11.52 & 115.09 & 56.33 & 13.99 & 176.25 & 84.92 \\
\hline & Median & 8.02 & 29.09 & 14.10 & 10.22 & 65.95 & 31.79 & 11.39 & 116.45 & 54.93 & 13.49 & 175.18 & 84.75 \\
\hline \multirow{2}{*}{$1,3 \mathrm{nr}_{\min }$} & Mean & 8.76 & 28.07 & 15.23 & 9.27 & 64.36 & 33.07 & 11.09 & 115.49 & 61.59 & 13.71 & 178.14 & 97.69 \\
\hline & Median & 8.92 & 28.43 & 15.09 & 9.24 & 67.37 & 35.52 & 11.56 & 117.29 & 64.20 & 13.17 & 181.84 & 98.12 \\
\hline \multirow{2}{*}{$1,2 \mathrm{nr}_{\min }$} & Mean & 10.00 & 26.48 & 12.82 & 9.75 & 62.89 & 33.37 & 11.92 & 117.38 & 65.73 & 12.60 & 181.64 & 101.39 \\
\hline & Median & 8.57 & 26.46 & 13.50 & 9.75 & 66.18 & 36.33 & 10.69 & 119.35 & 67.35 & 12.95 & 182.74 & 106.27 \\
\hline \multirow{2}{*}{$1,1 \mathrm{nr}_{\min }$} & Mean & 8.28 & 25.02 & 12.69 & 10.32 & 59.88 & 33.02 & 12.01 & 112.35 & 63.18 & 13.47 & 171.76 & 100.77 \\
\hline & Median & 9.01 & 24.42 & 13.15 & 10.31 & 62.32 & 34.66 & 11.30 & 117.89 & 66.65 & 12.97 & 176.77 & 108.11 \\
\hline \multirow{2}{*}{$\mathrm{nr}_{\text {min }}$} & Mean & 9.24 & 21.11 & 11.85 & 10.76 & 56.78 & 32.94 & 12.02 & 104.63 & 61.19 & 13.88 & 171.95 & 96.88 \\
\hline & Median & 9.07 & 20.27 & 12.36 & 10.11 & 60.73 & 33.05 & 11.50 & 109.02 & 63.68 & 14.69 & 173.87 & 103.69 \\
\hline
\end{tabular}

reductions in CPU time, where reductions decrease as grid size increases. The running time of SAC scales roughly linearly with the grid size as shown in Figure 8. For a given grid size, running time for different router numbers varies by less than $40 \%$.

Standard Deviation and IQR for CPU times of algorithms are presented in Table 6. IQR and StdDev for SA is roughly twice that of SAC. So, in terms of robustness as well as mean running time, the SAC is markedly superior to SA.

We have supposed at the beginning that the number of routers is unknown. So, an implicit objective was to determine an optimal number of routers that can provide a good coverage. From simulation, first, there was no need to use an initial number of routers greater than $1.5 \mathrm{Nr} \_$min, since this number provided a coverage percentage of $100 \%$ with SAC algorithm. Secondly, $1.3 \mathrm{Nr} \_$min can be considered as a good compromise, since SAC algorithm can achieve a coverage percentage greater than $99 \%$ with this number of routers. This algorithm improves results previously achieved in the literature by SA algorithm which is $98 \%$ using 1.3Nr_min routers (Fendji et al., 2016), and outperforms the approach based on metropolis proposed earlier in (Fendji et al., 2014).

Figure 9-12 presents the coverage of area of interest and the network topology achieved by SAC approach using $1.3 \mathrm{nr}_{\min }$ on four instances of different sizes. We can observe that the great percentage of EAI is covered by only one router, while the percentage covered by two routers is low and the percentage covered by three is almost zero. 
Table 6. Comparison of standard deviation and IQR CPU times of algorithms

\begin{tabular}{|c|c|c|c|c|c|c|c|c|c|c|c|c|c|}
\hline \multirow{2}{*}{$\begin{array}{c}\text { Number } \\
\text { of } \\
\text { Routers }\end{array}$} & & \multicolumn{3}{|c|}{$50 \times 50$} & \multicolumn{3}{|c|}{$100 \times 100$} & \multicolumn{3}{|c|}{$150 \times 150$} & \multicolumn{3}{|c|}{$200 \times 200$} \\
\hline & & CM & SA & SAC & CM & SA & SAC & CM & SA & SAC & $\mathbf{C M}$ & SA & SAC \\
\hline \multirow{2}{*}{$1,5 \mathrm{nr}_{\min }$} & Dev & 3.15 & 6.71 & 2.83 & 3.84 & 10.43 & 4.17 & 4.45 & 15.33 & 6.43 & 4.02 & 20.33 & 10.14 \\
\hline & IQR & 4.10 & 8.44 & 3.38 & 5.09 & 13.41 & 5.16 & 6.66 & 20.45 & 8.30 & 5.72 & 23.22 & 13.28 \\
\hline \multirow{2}{*}{$1,4 \mathrm{nr}_{\min }$} & Dev & 2.99 & 6.07 & 3.38 & 3.25 & 10.72 & 5.85 & 4.25 & 16.58 & 8.67 & 4.53 & 23.21 & 12.02 \\
\hline & IQR & 4.22 & 5.32 & 3.76 & 4.47 & 16.23 & 7.22 & 5.86 & 16.98 & 11.92 & 6.97 & 25.61 & 17.35 \\
\hline \multirow{2}{*}{$1,3 \mathrm{nr}_{\min }$} & Dev & 3.42 & 4.23 & 3.76 & 3.71 & 11.94 & 5.93 & 4.22 & 15.51 & 10.83 & 3.93 & 21.00 & 14.17 \\
\hline & IQR & 4.77 & 7.03 & 4.77 & 4.47 & 13.11 & 7.45 & 5.30 & 21.91 & 14.89 & 4.39 & 29.75 & 19.56 \\
\hline \multirow{2}{*}{$1,2 \mathrm{nr}_{\min }$} & Dev & 3.36 & 5.07 & 2.79 & 3.41 & 10.56 & 6.58 & 4.10 & 16.38 & 10.64 & 3.94 & 19.79 & 14.33 \\
\hline & IQR & 4.13 & 6.97 & 4.02 & 4.68 & 13.25 & 8.79 & 4.54 & 20.14 & 12.62 & 5.90 & 26.08 & 22.35 \\
\hline \multirow{2}{*}{$1,1 \mathrm{nr}_{\min }$} & Dev & 3.11 & 5.31 & 2.97 & 4.09 & 9.90 & 6.28 & 3.43 & 16.75 & 11.96 & 4.14 & 21.04 & 15.57 \\
\hline & IQR & 4.38 & 9.53 & 3.23 & 4.91 & 14.40 & 9.05 & 4.65 & 20.15 & 14.14 & 5.42 & 20.79 & 21.64 \\
\hline \multirow{2}{*}{$\mathrm{nr}_{\text {min }}$} & Dev & 3.22 & 3.53 & 2.75 & 3.84 & 9.96 & 7.38 & 4.17 & 16.11 & 12.75 & 4.95 & 19.81 & 14.08 \\
\hline & IQR & 4.25 & 4.86 & 3.28 & 4.76 & 13.51 & 9.06 & 5.69 & 19.40 & 14.97 & 6.71 & 22.46 & 21.89 \\
\hline
\end{tabular}

Figure 8. Average CPU time used by the different algorithms

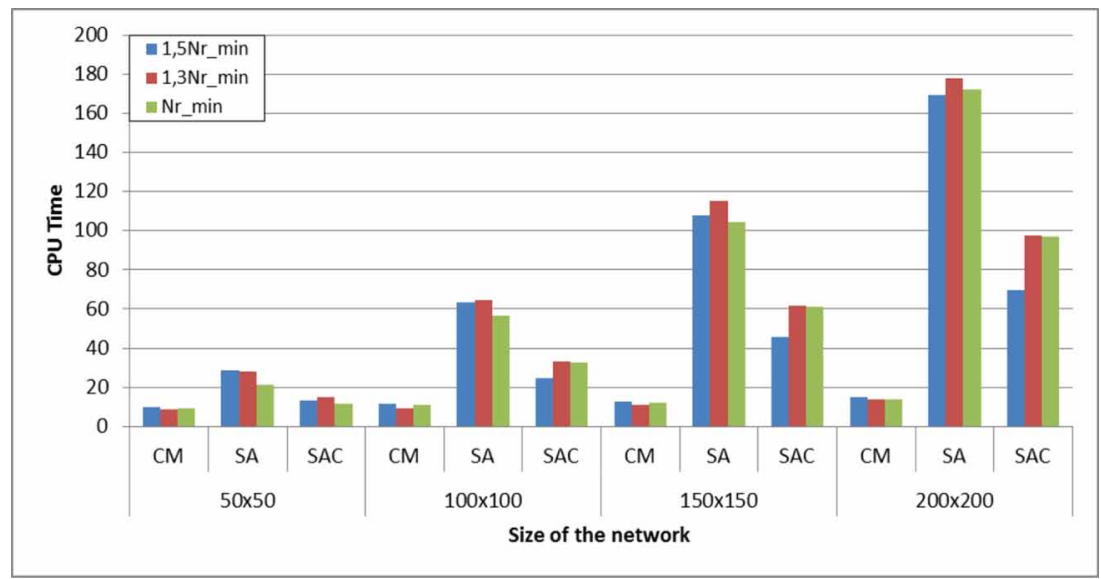

\section{CONCLUSION AND FURTHER WORK}

In this paper, a new algorithm for node placement in rural wireless mesh networks is proposed. Simulated Annealing based Centre of Mass (SAC) combines the best features of the Centre of Mass (CM) and Simulated Annealing (SA) algorithms. CM is a descent algorithm which converges quickly to a local optimum; while SA employs stochasticity to explore a larger area of the solution space. SAC combines both in such a way as to preserve the exploration properties of SA, while employing $\mathrm{CM}$ characteristics to more effectively seek out local optima in the regions explored.

SAC achieved better performance than previous algorithms. 99\% coverage was achieved when router number $=1.3 \mathrm{nr}_{\text {min }}$, which surpasses the $98 \%$ found in (Fendji et al., 2016), and $95 \%$ found in (Fendji et al., 2015; Fendji et al., 2014).

Simulation results on 24 benchmark instances show that SAC consistently achieves much better average coverage than CM for all grid sizes $(\mathrm{p}<0.00001)$, and also better coverage than $\mathrm{SA}$ for larger grid sizes $(\mathrm{p}<0.00001)$. In addition, compared with $\mathrm{SA}$, SAC both reduces the 
Figure 9. (a) Router coverage and (b) Network Topology provided by SAC approach on a $50 \times 50$ Instance using $1.3 \mathrm{nr}$ _min routers. Blue areas are covered by one router, red areas by two, and white areas by three routers.

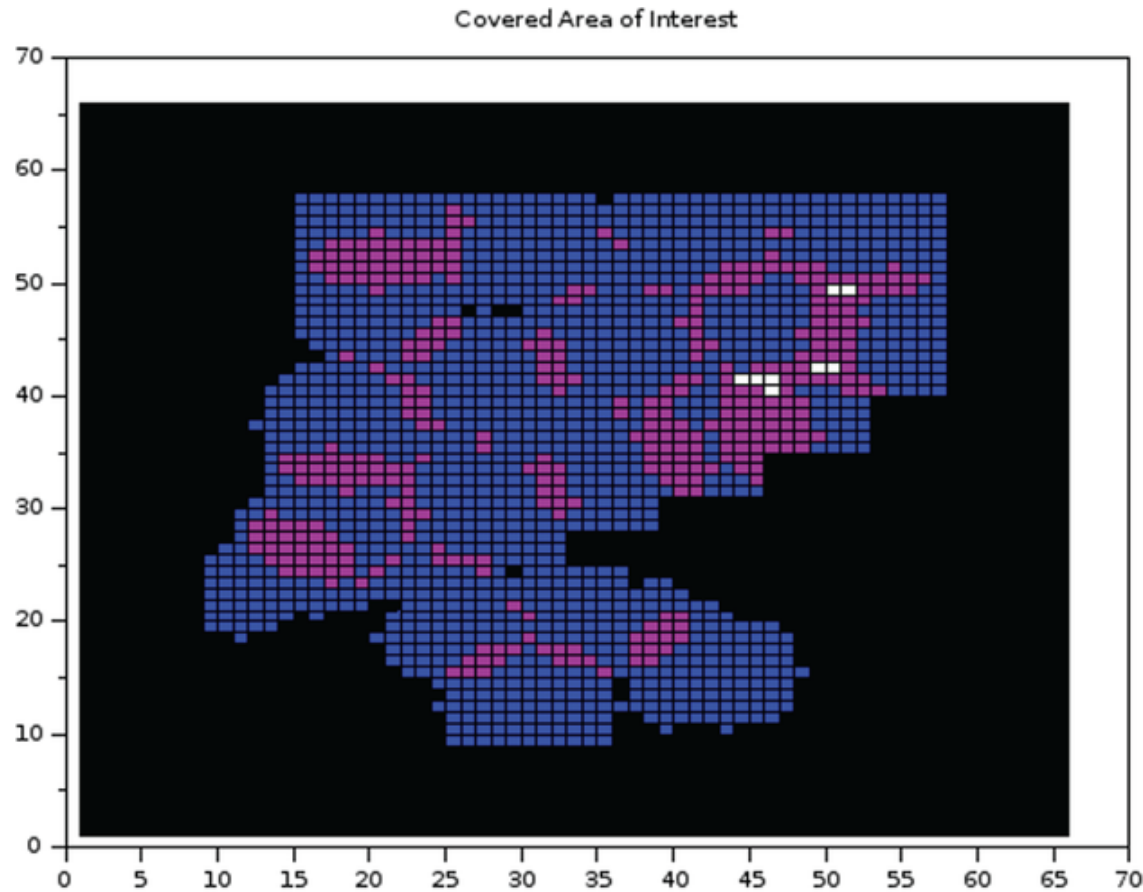

(a)

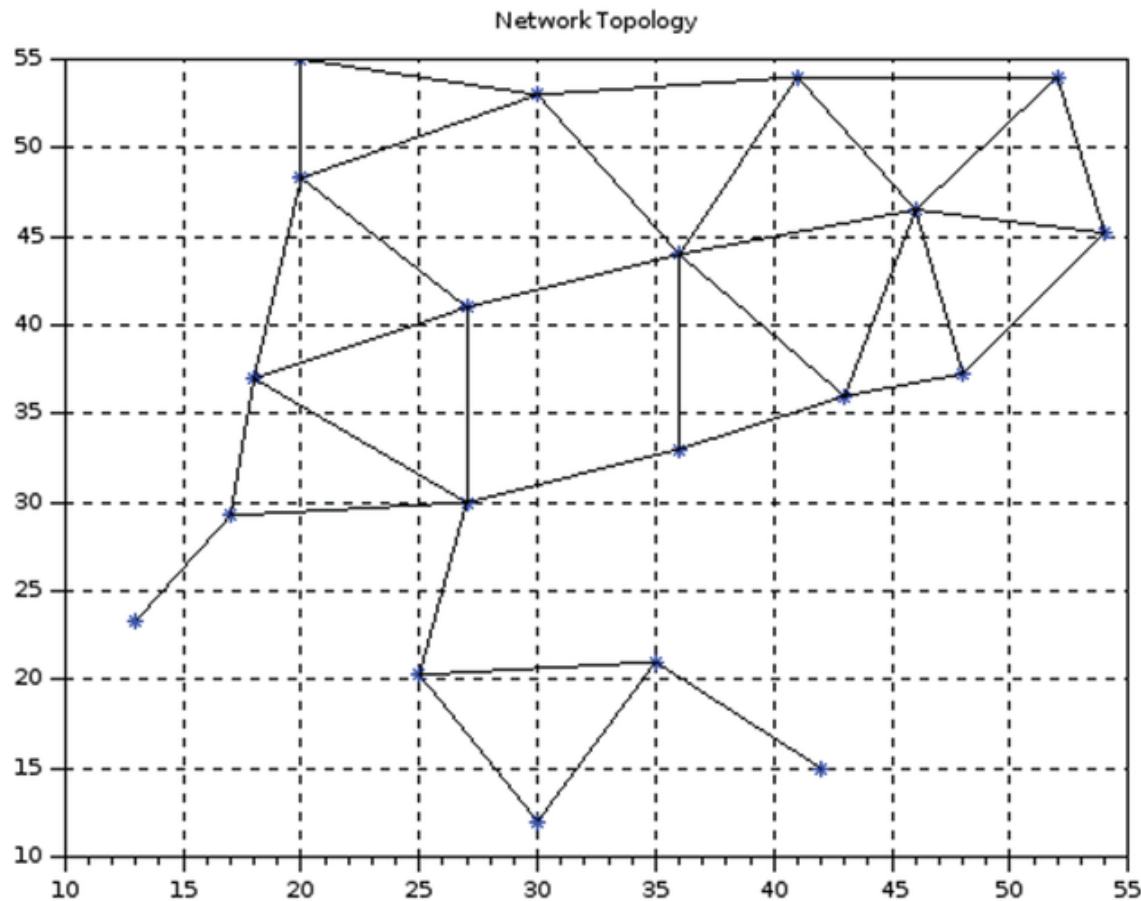

(b) 
Figure 10. (a) Router coverage and (b) Network Topology provided by SAC approach on a 100x100 Instance using 1.3nr_min routers. Blue areas are covered by one router, red areas by two, and white areas by three routers.

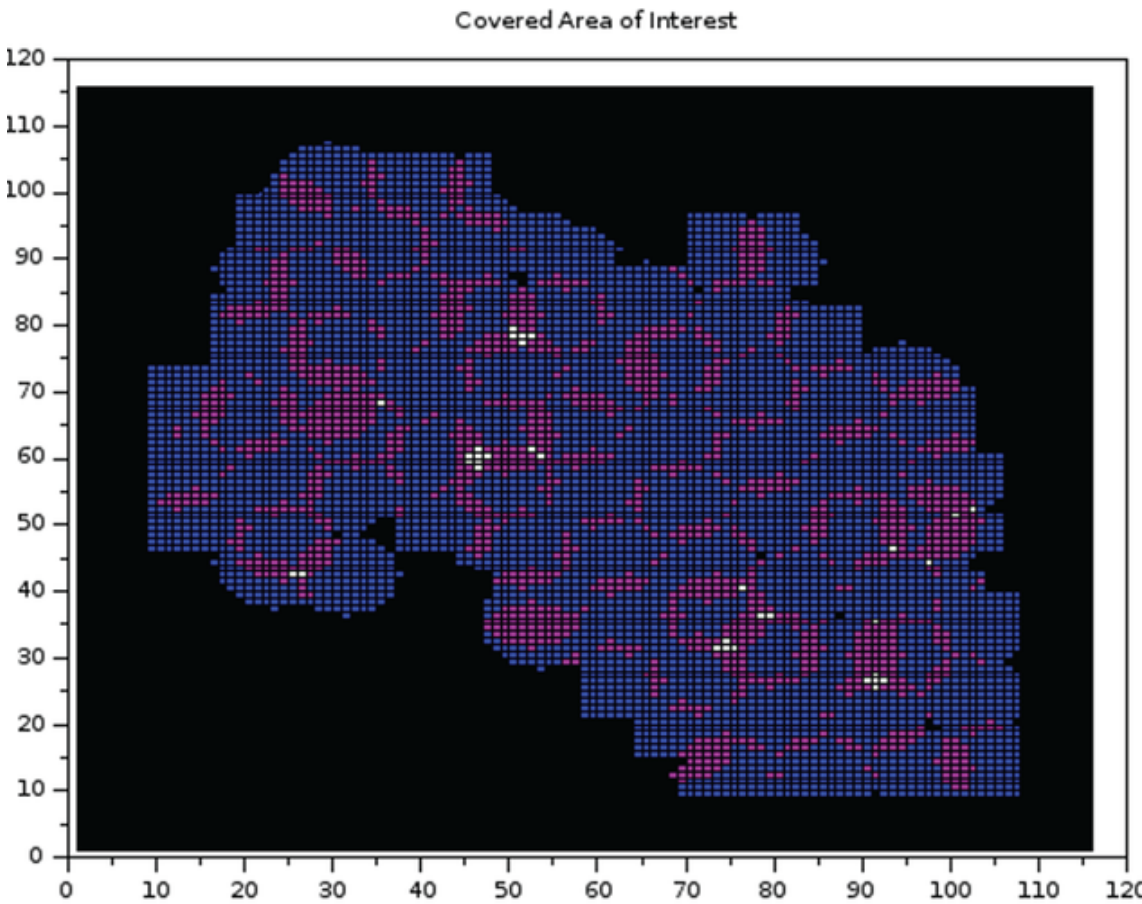

(a)



(b) 
Figure 11. (a) Router coverage and (b) Network Topology provided by SAC approach on a 150x150 Instance using 1.3nr_min routers. Blue areas are covered by one router, red areas by two, and white areas by three routers.

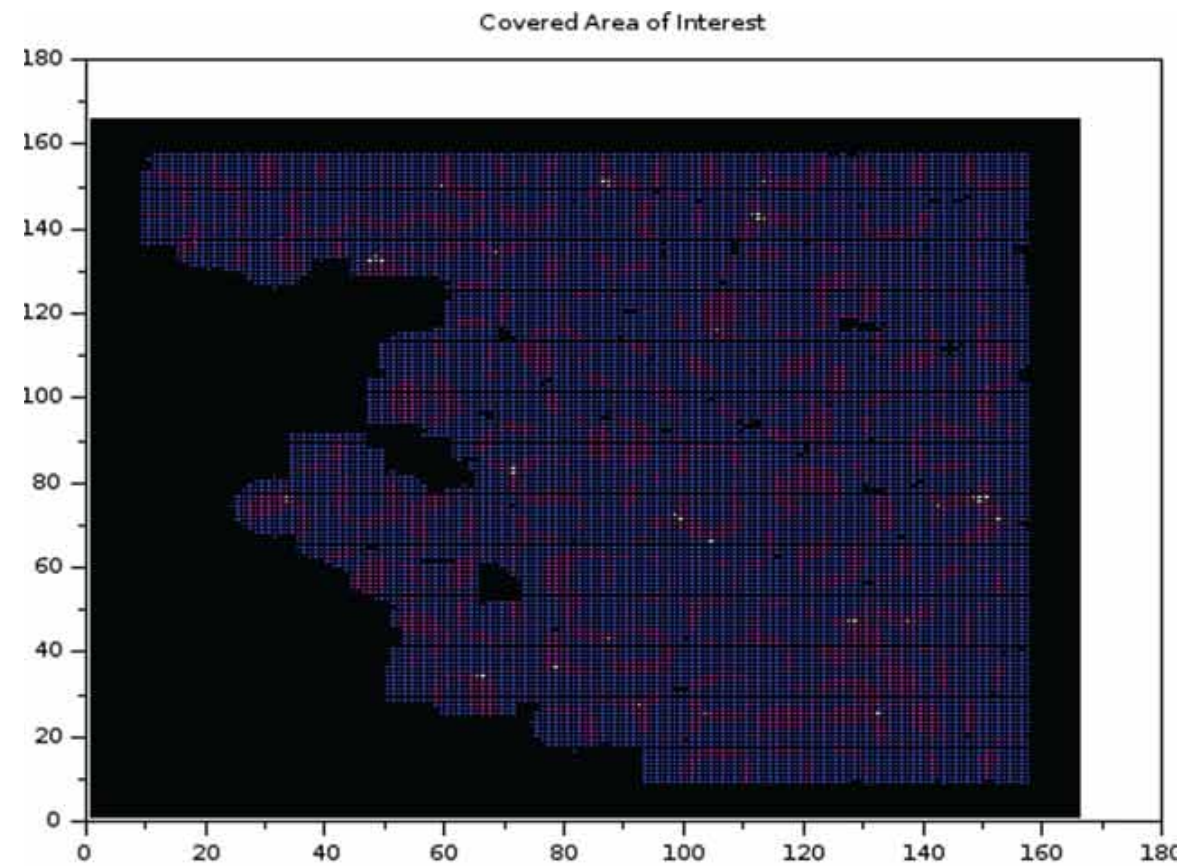

(a)

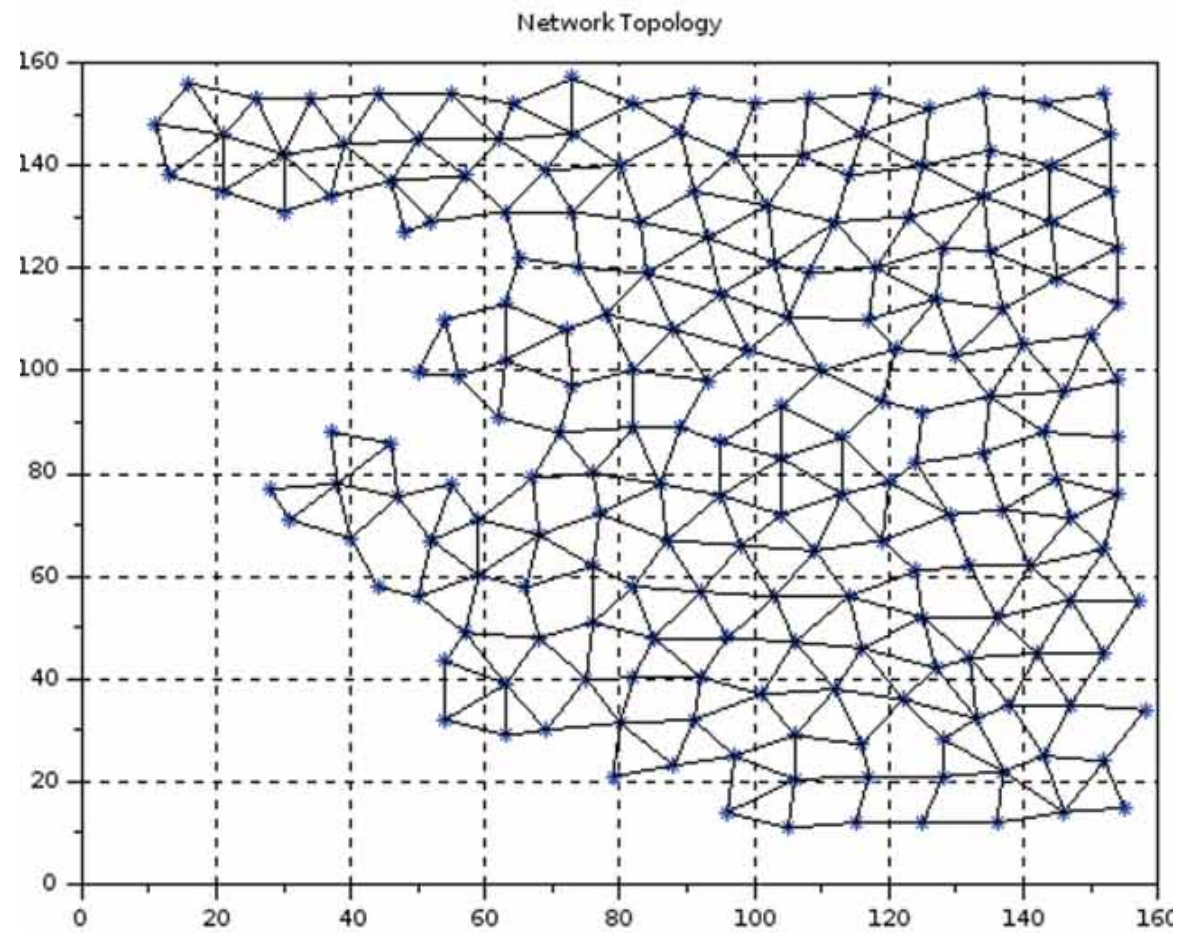

(b) 
Figure 12. (a) Router coverage and (b) Network Topology provided by SAC approach on a $200 \times 200$ Instance using 1.3nr_min routers. Blue areas are covered by one router, red areas by two, and white areas by three routers.

Covered Area of Interest

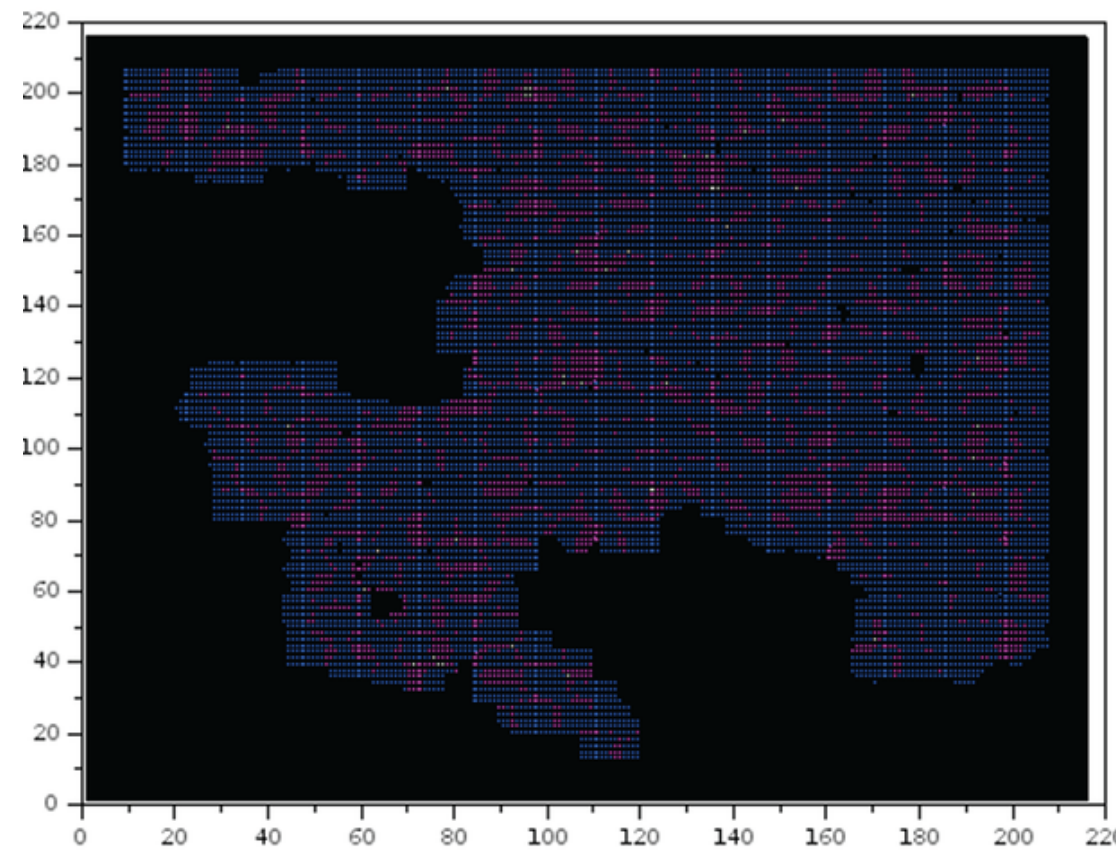

(a)

Network Topology



(b) 
running time (for all grid sizes) and improves the coverage robustness by reducing the variance of solutions (for larger grid sizes).

The above results indicate that SAC holds promise as a high-performing algorithm for practical design of rural WMN's. On a managerial point of view, SAC's contribution is twofold. First, SAC reduces the time consumed during the network planning phase. Secondly, SAC increases the network coverage percentage for a given number of routers. That means, for the same coverage percentage $\mathrm{SAC}$ will require fewer nodes (reducing the cost of the architecture), or for a given number of nodes, SAC will provide a greater network coverage (improving the network coverage).

Future work may include application of the algorithm to areas of interest with different characteristics, including non-continuous areas. Additionally, the question of guaranteeing good connectivity has not been addressed: this issue is particularly important in areas of interest with significant obstacles that may block communication between mesh routers. Modified algorithms may be developed to address this issue by ensuring bi- or tri-connectivity of the network, which increases robustness of the functioning network. Finally, the SAC approach may be made more efficient by developing a more intelligent strategy for router moves in the SA subsystem of SAC, to replace the purely random moves employed in the current algorithm. 


\section{REFERENCES}

Akyildiz, I. F., \& Wang, X. (2008). Cross-layer design in wireless mesh networks. IEEE Transactions on Vehicular Technology, 57(2), 1061-1076. doi:10.1109/TVT.2007.911615

Akyildiz, I. F., Wang, X., \& Wang, W. (2005). Wireless mesh networks: A survey. Computer Networks, 47(4), 445-487. doi:10.1016/j.comnet.2004.12.001

Amaldi, E., Capone, A., Cesana, M., Filippini, I., \& Malucelli, F. (2008). Optimization models and methods for planning wireless mesh networks. Computer Networks, 52(11), 2159-2171. doi:10.1016/j.comnet.2008.02.020

Aoun, B., Boutaba, R., Iraqi, Y., \& Kenward, G. (2006). Gateway Placement Optimization in Wireless Mesh Networks With QoS Constraints. IEEE Journal on Selected Areas in Communications, 24(11), 2127-2136. doi:10.1109/JSAC.2006.881606

Backens, J., Mweemba, G., \& Van Stam, G. (2009, December). A rural implementation of a 52 node mixed wireless mesh network in Macha, Zambia. Proceedings of the International Conference on e-Infrastructure and e-Services for Developing Countries (pp. 32-39). Springer. doi:10.1007/978-3-642-12701-4_4

Beljadid, a., Hafid, a. S., \& Gendreau, M. (2007). Optimal Design of Broadband Wireless Mesh Networks. Proceedings of the IEEE GLOBECOM 2007 - IEEE Global Telecommunications Conference, (4840), 4840-4845. doi:10.1109/GLOCOM.2007.918

Benyamina, D., Hafid, A., \& Gendreau, M. (2008). A multi-objective optimization model for planning robust and least interfered wireless mesh networks. Proceedings of the GLOBECOM - IEEE Global Telecommunications Conference. IEEE. doi:10.1109/GLOCOM.2008.ECP.1014

Benyamina, D., Hafid, A., \& Gendreau, M. (2008). Wireless mesh network planning: A multi-objective optimization approach. Proceedings of the 5 th International Conference on Broadband Communications, Networks and Systems BROADNETS 2008 (pp. 602-609). Academic Press. doi:10.1109/BROADNETS.2008.4769149

Benyamina, D., Hafid, A., \& Gendreau, M. (2009). Optimal placement of gateways in multi-hop wireless mesh networks: A clustering-based approach. Proceedings of the Conference on Local Computer Networks (pp. 625-632). Academic Press. doi:10.1109/LCN.2009.5355141

Benyamina, D., Hafid, A., \& Gendreau, M. (2012). Wireless Mesh Networks Design \#x2014; A Survey. IEEE Communications Surveys and Tutorials, 14(2), 299-310. doi:10.1109/SURV.2011.042711.00007

Benyamina, D., Hallam, N., \& Hafid, A. (2008). On optimizing the planning of multi-hop wireless networks using a multi objective evolutionary approach. International Journal of Communication, 2(4), 213-221. Retrieved from http://citeseerx.ist.psu.edu/viewdoc/download?doi=10.1.1.462.810\&rep=rep1\&type $=$ pdf

Bernardi, G., Marina, M. K., Talamona, F., \& Rykovanov, D. (2011). IncrEase: A tool for incremental planning of rural fixed Broadband Wireless Access networks. Proceedings of the 2011 IEEE GLOBECOM Workshops (GC Wkshps) (pp. 1013-1018). IEEE. doi:10.1109/GLOCOMW.2011.6162330

Chaudhry, A. U., Hafez, R. H. M., Aboul-Magd, O., \& Mahmoud, S. A. (2010). Throughput improvement in multi-radio multi-channel 802.11a-based wireless mesh networks. Proceedings of the GLOBECOM - IEEE Global Telecommunications Conference. IEEE. doi:10.1109/GLOCOM.2010.5684193

Chen, C., \& Chekuri, C. (2007). Urban Wireless Mesh Network Planning: The Case of Directional Antennas.

Chen, S.-M., Chien, C.-Y., Chena, S.-M., \& Chiena, C.-Y. (2011). Solving the traveling salesman problem based on the genetic simulated annealing ant colony system with particle swarm optimization techniques. Expert Systems with Applications, 38(12), 14439-14450. doi:10.1016/j.eswa.2011.04.163

Darties, B., Theoleyre, F., \& Duda, A. (2009). A divide-and-conquer scheme for assigning roles in multi-channel wireless mesh networks. Proceedings - Conference on Local Computer Networks (pp. 277-280). Academic Press. doi:10.1109/LCN.2009.5355087

Das, A. K., Alazemi, H. M. K., Vijayakumar, R., \& Roy, S. (2005). Optimization models for fixed channel assignment in wireless mesh networks with multiple radios. Proceedings of the 2005 Second Annual IEEE Communications Society Conference on Sensor and Ad Hoc Communications and Networks IEEE SECON 2005 (pp. 463-474). IEEE. 
De Marco, G. (2009). MOGAMESH: A multi-objective algorithm for node placement in wireless mesh networks based on genetic algorithms. Proceedings of the 2009 6th International Symposium on Wireless Communication Systems (pp. 388-392). IEEE. doi:10.1109/ISWCS.2009.5285321

Ezugwu, A. E.-S., Adewumi, A. O., \& Frîncu, M. E. (2017). Simulated annealing based symbiotic organisms search optimization algorithm for traveling salesman problem. Expert Systems with Applications, 77, 189-210. doi:10.1016/j.eswa.2017.01.053

Fendji, J.L.E.K. (2017). Instances for Simulated Annealing based Centre of Mass. doi:10.17632/3VBJXPCS6P.1

Fendji, J. L. E. K., Thron, C., \& Nlong, J. M. (2016). Simulated annealing approach for mesh router placement in rural wireless mesh networks. Proceedings of the International Conference on e-Infrastructure and e-Services for Developing Countries (pp. 192-202). Springer. doi:10.1007/978-3-319-43696-8_19

Fendji Kedieng Ebongue, J. L., Thron, C., \& Nlong, J. M. (2014). Mesh Router Nodes placement in Rural Wireless Mesh Networks. Proceedings of the CARI Conference. Academic Press.

Fendji Kedieng Ebongue, J. L., Thron, C., \& Nlong, J. M. (2015). A Metropolis Approach for Mesh Router Nodes placement in Rural Wireless Mesh Networks. Journal of Computers, 10(2), 101-114. doi:10.17706/ jcp.10.2.101-114

Franklin, A., \& Murthy, C. S. R. (2007). Node Placement Algorithm for Deployment of Two-Tier Wireless Mesh Networks. IEEE GLOBECOM 2007-2007 IEEE Global Telecommunications Conference, 4823-4827. doi:10.1109/GLOCOM.2007.915

Fu, B., Xiao, Y., Deng, H. J., \& Zeng, H. (2014). A survey of cross-layer designs in wireless networks. IEEE Communications Surveys and Tutorials, 16(1), 110-126. doi:10.1109/SURV.2013.081313.00231

Garces, R., \& Garcia-Luna-Aceves, J. J. (2000). Collision avoidance and resolution multiple access for multichannel $\backslash n$ wireless networks. Proceedings IEEE INFOCOM 2000. Academic Press. doi:10.1109/ INFCOM.2000.832233

Gupta, P., \& Kumar, P. R. (2000). The capacity of wireless networks. IEEE Transactions on Information Theory, 46(2), 388-404. doi:10.1109/18.825799

Ingber, L. (1989). Very fast simulated re-annealing. Mathematical and Computer Modelling, 12(8), 967-973. doi:10.1016/0895-7177(89)90202-1

Jia, Z. Y., Ma, J. W., Wang, F. J., \& Liu, W. (2011). Hybrid of simulated annealing and SVM for hydraulic valve characteristics prediction. Expert Systems with Applications, 38(7), 8030-8036. doi:10.1016/j.eswa.2010.12.132

Jingzhi, D., Jianxiao, X., \& Zhifeng, Z. (2009). Gateway Deployment Optimization in Wireless Mesh Network: A Case Study in China. Proceedings of the 2009 IEEE/INFORMS International Conference on Service Operations, Logistics and Informatics. IEEE. doi:10.1109/SOLI.2009.5203949

Kirkpatrick, S. S., Gelatt, C. D. C. D., \& Vecchi, M. P. M. P. (1983). Optimization by simulated annealing. Science, 220(4598), 671-680. doi:10.1126/science.220.4598.671 PMID:17813860

Liu, T., \& Liao, W. (2009). Interference-aware QoS routing for multi-rate multi-radio multi-channel IEEE 802.11 wireless mesh networks. IEEE Transactions on Wireless Communications, 8(1), 166-175. doi:10.1109/TWC.2009.070369

Marina, M. K., Das, S. R., \& Subramanian, A. P. (2010). A topology control approach for utilizing multiple channels in multi-radio wireless mesh networks. Computer Networks, 54(2), 241-256. doi:10.1016/j. comnet.2009.05.015

Metropolis, N., Rosenbluth, A. W., Rosenbluth, M. N., \& Teller, A. H. (1953). Metropolis. The Journal of Chemical Physics, 21(6), 1087-1092. doi:10.1063/1.1699114

Pathak, P. H., \& Dutta, R. (2011). A Survey of Network Design Problems and Joint Design Approaches in Wireless Mesh Networks. IEEE Communications Surveys and Tutorials, 13(3), 396-428. doi:10.1109/ SURV.2011.060710.00062 
Roh, H. T., \& Lee, J. W. (2010). Optimal placement of a relay node with controllable mobility in wireless networks considering fairness. Proceedings of the 2010 7th IEEE Consumer Communications and Networking Conference, CCNC 2010. IEEE. doi:10.1109/CCNC.2010.5421793

Sen, S., \& Raman, B. (2007). Long distance wireless mesh network planning: problem formulation and solution (pp. 893-902). New York: ACM; doi:10.1145/1242572.1242693

Talbi, E. G. (2009). Metaheuristics: From Design to Implementation. Metaheuristics: From Design to Implementation. John Wiley \& Sons. doi:10.1002/9780470496916

Tang, M. (2009). Gateways Placement in Backbone Wireless Mesh Networks. International Journal of Communications. Network and System Sciences, 2(1), 44-50. doi:10.4236/ijcns.2009.21005

Varanelli, J. M., \& Cohoon, J. P. (1999). A fast method for generalized starting temperature determination in homogeneous two-stage simulated annealing systems. Computers \& Operations Research, 26(5), 481-503. doi:10.1016/S0305-0548(98)00062-8

Wang, F., Thai, M. T., \& Du, D. Z. (2009). On the construction of 2-connected virtual backbone in wireless networks. IEEE Transactions on Wireless Communications, 8(3), 1230-1237. doi:10.1109/TWC.2009.051053

Wang, J., Xie, B., Cai, K., \& Agrawal, D. P. (2007, October). Efficient mesh router placement in wireless mesh networks. Proceedings of the 2007 IEEE International Conference on Mobile Adhoc and Sensor Systems (pp. 1-9). IEEE. doi:10.1109/MOBHOC.2007.4428616

Wu, W., Luo, J., \& Yang, M. (2009). Gateway placement optimization for load balancing in wireless mesh networks. Proceedings of the 2009 13th International Conference on Computer Supported Cooperative Work in Design (pp. 408-413). IEEE. doi:10.1109/CSCWD.2009.4968093

Wu, W., Luo, J., \& Yang, M. (2010). Cost-effective placement of mesh nodes in wireless mesh networks. Proceedings of the 5th International Conference on Pervasive Computing and Applications (pp. 261-266). IEEE. doi:10.1109/ICPCA.2010.5704109

Xhafa, F., Barolli, A., Sánchez, C., \& Barolli, L. (2011). A simulated annealing algorithm for router nodes placement problem in wireless mesh networks. Simulation Modelling Practice and Theory, 19(10), 2276-2284. doi:10.1016/j.simpat.2010.08.014

Xhafa, F., Sanchez, C., \& Barolli, L. (2009). Ad Hoc and Neighborhood Search Methods for Placement of Mesh Routers in Wireless Mesh Networks. Proceedings of the 200929 th IEEE International Conference on Distributed Computing Systems Workshops (pp. 400-405). IEEE. doi:10.1109/ICDCSW.2009.37

Xhafa, F., Sánchez, C., \& Barolli, L. (2010). Genetic algorithms for efficient placement of router nodes in wireless mesh networks. Proceedings - International Conference on Advanced Information Networking and Applications, AINA (pp. 465-472). Academic Press. doi:10.1109/AINA.2010.41

Xin, Q., \& Wang, Y. J. (2009). Gateway Selection Scheme for Throughput Optimization in Multi-radio Multichannel Wireless Mesh Networks. Proceedings of the 2009 Fifth International Conference on Mobile Ad-hoc and Sensor Networks (pp. 187-195). IEEE. doi:10.1109/MSN.2009.42

Xu, L., Yamamoto, K., Murata, H., \& Yoshida, S. (2008). Performance improvement of wireless mesh networks by using a combination of channel-bonding and multi-channel techniques. IEICE Transactions on Communications, 91(10), 3103-3112.

Yannibelli, V., \& Amandi, A. (2013). Hybridizing a multi-objective simulated annealing algorithm with a multiobjective evolutionary algorithm to solve a multi-objective project scheduling problem. Expert Systems with Applications, 40(7), 2421-2434. doi:10.1016/j.eswa.2012.10.058

Yuping, Z., Shouwei, J., \& Chunli, Z. (2005). A very fast simulated re-annealing algorithm for the leather nesting problem. International Journal of Advanced Manufacturing Technology, 25(11-12), 1113-1118. doi:10.1007/ s00170-003-1966-6

Zhou, P., Wang, X., Manoj, B. S., \& Rao, R. (2010). On optimizing gateway placement for throughput in wireless mesh networks. EURASIP Journal on Wireless Communications and Networking, 2010(1), 368423. doi:10.1155/2010/368423 
Fendji Kedieng Ebongue Jean Louis was born in Douala, Cameroon, in 1986. He received the B.Sc. and M.SC. degrees in computer science from University of Ngaoundéré, Cameroon, in 2007 and 2010, respectively, and his PhD from the University of Bremen, Germany, in 2015. He has been working as a scientist in the BMBF-Project CMR 10/P01 between the University of Ngaoundéré and the University of Bremen (2011-2013). He is currently a lecturer at the University of Ngaoundéré. Current research interests focus on optimisation techniques for the design of sustainable networks and services.

Christopher Thron is associate professor of mathematics at Texas A\&M University-Central Texas. Formerly, he was a system engineer with Freescale Semiconductor. He has PhDs from the University of Wisconsin and the University of Kentucky. His focus areas include machine learning, operations research, optimization, agent-based modeling and algorithm design. Applications include signal processing, operations research, modeling, and public health statistics. He has a long-standing interest in Third World development, and has taught courses and workshops in mathematics, statistics, and software in Benin, Cameroon, Chad, Nigeria, Sudan, and China. He has also published research with faculty and other experts from those countries. 